\title{
Tree-based Group Key Agreement *
}

\author{
Yongdae $\mathrm{Kim}^{1}$, Adrian Perrig ${ }^{2}$, and Gene Tsudik ${ }^{3}$ \\ 1 Computer Science and Engineering Department \\ University of Minnesota - Twin Cities \\ kydecs.umn.edu \\ 2 Electrical and Computer Engineering Department \\ Carnegie Mellon University \\ perrig@cmu.edu \\ 3 Department of Information and Computer Science, \\ University of California at Irvine \\ gts@ics.uci.edu
}

\begin{abstract}
Secure and reliable group communication is an active area of research. Its popularity is caused by the growing importance of group-oriented and collaborative applications. The central research challenge is secure and efficient group key management. While centralized methods are often appropriate for key distribution in large multicast-style groups, many collaborative group settings require distributed key agreement techniques. This work investigates a novel group key agreement approach which blends so-called key trees with Diffie-Hellman key exchange. It yields a secure protocol suite (TGDH) that is both simple and fault-tolerant. Moreover, the efficiency of TGDH appreciably surpasses that of prior art.
\end{abstract}

\section{Introduction}

Fault-tolerant, scalable, and reliable communication services have become critical in modern computing. An important and popular trend is to convert traditional centralized services (e.g., file sharing, authentication, web, and mail) into distributed services spread across multiple systems and networks. Many of these newly distributed and other inherently collaborative applications (e.g., conferencing, white-boards, shared instruments, and command-and-control systems) need secure communication. However, experience shows that security mechanisms for collaborative and dynamic peer groups tend to be both expensive and unexpectedly complex. In that regard, dynamic peer groups are very different from non-collaborative, centrally managed, one-to-many (or few-to-many) broadcast groups such as those encountered in Internet multicast.

Dynamic Peer Groups (DPGs) are common in many layers of the network protocol stack and many application areas of modern computing. Examples of DPGs include replicated servers (such as database, web, time), audio and video conferencing and, more generally, applications supporting collaborative work. In contrast to large multicast groups, DPGs tend to be relatively small in size, on the order of hundred members. Larger groups are harder to control on a peer basis and are often organized in a hierarchy. DPGs typically assume a many-to-many (or, equivalently, any-to-any) communication pattern rather than one-to-many pattern common of larger hierarchical groups.

Despite their relatively small number, group members in a DPG may be spread throughout the Internet and must be able to deal with arbitrary partitions due to network failures, congestion, and hostile attacks. In essence, a group can be split into a number of disconnected partitions each of which must persist and function as an independent peer group.

Security requirements in collaborative DPGs present several interesting research challenges. In this paper, we focus on secure and efficient group key management. The goal of group key management is to set up and maintain a shared secret key among the group members. It serves as a cornerstone for other DPG security services.

\subsection{Group Key Management}

There are several fundamentally different approaches to group key management in peer groups.

One approach relies on a single entity (called a key server) to generate keys and distribute them to the group. We refer to it as centralized group key distribution. Essentially, a key server maintains long-term shared keys with each

\footnotetext{
* An early version of this paper has appeared, in part, in [21].
} 
group member in order to enable secure two-party communication for the actual key distribution. One form of this solution uses a fixed trusted third party (TTP) as the key server. This approach has two problems: 1) TTP must be constantly available, and 2) TTP must exist in every possible subset of a group in order to support continued operation in the event of network partitions. The first problem can be addressed with fault-tolerance and replication techniques. The second is impossible to solve in a scalable and efficient manner. We note, however, that the centralized approach works well in one-to-many multicast scenarios since a TTP (or a set thereof) placed at, or very near, the source of communication can support continued operation within an arbitrary partition as long as it includes the source. Typically, one-to-many settings only aim to offer continued operation within a single partition that includes the source. Whereas, many-to-many environments must offer continued operation in an arbitrary number of partitions.

Another approach - called decentralized group key distribution - involves dynamically selecting a group member to generate and distribute keys to other group members. This approach is more robust and, thus, more applicable to many-to-many groups since any partition can continue operation by electing a key server. The drawback is that, as in the TTP case, a key server must establish long-term pairwise secure channels with all current group members in order to distribute group keys. Consequently, each time a new key server comes into play, significant costs must be incurred to set up these channels. Another disadvantage, again as in the TTP case, is the reliance on a single entity to generate good (i.e., cryptographically strong, random) keys.

In contrast to the above approaches, contributory group key management requires each group member to contribute an equal share to the common group key (which is then computed as a function of all members' contributions). This avoids the problems with the centralized trust and the single point of failure. Moreover, some contributory methods do not require the establishment of pairwise secret channels among group members. One significant problem with, current contributory group key agreement ${ }^{1}$ protocols is that they are not designed to tolerate failures and group membership changes during execution. In particular, nested (cascaded) failures, partitions and other group events are not accommodated. This is not surprising since most multi-round cryptographic protocols do not offer built-in robustness with the notable exception of protocols for fair exchange [6].

\subsection{Overview}

In this paper, we focus on contributory group key agreement. In doing so, we unify two important trends in group key management: 1) key trees to efficiently compute and update group keys and 2) Diffie-Hellman key exchange to achieve provably secure and fully distributed protocols. Our main result is a simple, secure, robust and efficient key management solution, called TGDH (Tree-based Group Diffie-Hellman).

Organization: The rest of this paper is organized as follows. Section 2 presents our assumptions and requirements for the reliable group communication system. Section 3 introduces cryptographic requirements of our group key agreement protocol and Section 4 introduces notation and terminology. The actual protocols are described in Section 5 followed by practical aspects of the protocol in Section 6. Section 7 analyzes both conceptual and experimental protocol complexity. The summary of related work appears in Section 8. Finally, security argument of the proposed protocols are provided in Appendix A.

\section{Group Communication and Group Key Agreement}

As noted in the introduction, many modern collaborative and distributed applications require a reliable group communication platform. The latter, in turn, needs specialized security mechanisms to perform - among other things group key management. This dependency is mutual since practical group key agreement protocols themselves rely on the underlying group communication semantics for protocol message transport and strong membership semantics. Implementing multi-party and multi-round cryptographic protocols without such support is foolhardy as, in the end, one winds up reinventing reliable group communication tools.

In this section we begin with a brief discussion of reliable group communication. Next, we summarize the relationship between group membership events and group key management protocols and conclude with the summary of desired cryptographic properties.

\footnotetext{
${ }^{1}$ We use the term "agreement," as opposed to "distribution", to emphasize the contributory nature of the key management.
} 


\subsection{Group Communication Semantics and Support}

There are two commonly used strong group communication semantics: Extended Virtual Synchrony (EVS) [25, 2] and View Synchrony (VS) [19]. Both guarantee that: 1) group members see the same set of messages between two sequential group membership events, and, 2) sender's requested message order (e.g., FIFO, Causal, or Total) is preserved. VS provides a stricter service whereas EVS implementations are generally more efficient.

The main difference between EVS and VS is that EVS guarantees that messages are delivered to all receivers in the same membership as existed when the message was originally sent on the network. VS, in contrast, offers a stricter guarantee that messages are delivered to all recipients in the same membership as viewed by the sender application when it originally sent the message.

Providing the latter property requires an extra round of acknowledgment messages from all members before installing a new membership view. This need for acknowledgments dictates that the groups be closed, only allowing members of the group to send messages to it. However, the knowledge that a message is received in the membership the sender believed it was sent makes implementing secure group communication easier because every message is encrypted with the same key as the receiver believes is current when the message is delivered to them.

An implementation of any distributed fault-tolerant group key agreement protocol requires VS. This is because implementing group key agreement on top of EVS would require the key agreement protocol to incorporate and implement semantics identical to those of VS in order to correctly keep state of which messages were sent in which key epoch. (Intuitively, this is because membership events are unpredictable and each triggers an instance of a key agreement protocol. Thus, multiple key agreement protocols can overlap in time and cause instability unless significant amount of state is kept within the key agreement protocol implementation.) For this reason, there is no particular benefit to building key agreement on top of EVS semantics.

The issues surrounding implementation of key agreement in dynamic peer groups are addressed in detail in [3]. Suffice it to say that, in the context of this paper, we require the underlying group communication to provide View Synchrony (VS). However, we stress that VS is needed for the sake of fault-tolerance and robustness; the security of our protocols is in no way affected by the lack of VS.

\subsection{Group Membership Events}

A comprehensive group key agreement solution must handle adjustments to group secrets subsequent to all membership change operations in the underlying group communication system.

We distinguish among single and multiple member operations. Single member changes include member join or leave. Leave occurs when a member wants (or is forced) to leave a group. While there might be different reasons for member leave - such as voluntary leave, involuntary disconnect or forced expulsion - we believe that group key agreement must only provide the tools to adjust the group secrets and leave the rest up to the higher-layer (applicationdependent) security mechanisms.

Multiple member changes can also be additive and subtractive. We refer to the former operation as group merge, in which case two or more groups merge into a single group. We refer to the latter as group partition, whereby a group is split into smaller groups. A group partition can take place for several reasons two of which are fairly common:

1. Network failure - a network event causes disconnection within the group. Consequently, a group is split into fragments some of which are singletons while others (those that maintain mutual connectivity) are sub-groups.

2. Explicit (application-driven) partition - the application decides to split the group into multiple components or exclude multiple members at once.

Similarly, a group merge be either voluntary or involuntary:

1. Network fault heal - a network event causes previously disconnected network partitions to reconnect. Consequently, groups on all sides (and there might be more than two sides) of an erstwhile partition are merged into a single group.

2. Explicit (application-driven) merge - the application decides to merge multiple pre-existing groups into a single group. (The case of simultaneous multiple-member addition is not covered.)

At the first glance, events such as network partitions and fault heals might appear infrequent and dealing with them might seem a purely academic exercise. In practice, however, such events are common due to network misconfigurations and router failures. In [25], Moser et al. offer some compelling arguments in support of these claims. We consider coping with group partitions and merges to be a crucial component of group key agreement. 
In addition to the aforementioned membership operations, periodic refreshes of group secrets are advisable so as to limit the amount of ciphertext generated with the same key and to recover from potential compromises of members' contributions or prior session keys.

\section{Cryptographic Properties}

One of the most important security requirements of group key agreement is called key freshness. A key is fresh if it can be guaranteed to be new, as opposed to possibly an old key being reused an adversary.

Furthermore, a session key should be known only to the involved parties. We can now define four important security properties of group key agreement:

Definition 1. Assume that a group key is changed $m$ times and the sequence of successive group keys is $\mathcal{K}=$ $\left\{K_{0}, \ldots, K_{m}\right\}$.

1. Group Key Secrecy guarantees that it is computationally infeasible for a passive adversary to discover any group key $K_{i} \in \mathcal{K}$ for all $i$.

2. Forward Secrecy guarantees that a passive adversary who knows a contiguous subset of old group keys (say $\left\{K_{0}, K_{1}, \ldots, K_{i}\right\}$ ) cannot discover any subsequent group key $K_{j}$ for all $i$ and $j$ where $j>i$.

3. Backward Secrecy guarantees that a passive adversary who knows a contiguous subset group keys (say $\left.\left\{K_{i}, K_{i+1}, \ldots, K_{j}\right\}\right)$ cannot discover preceding group key $K_{l}$ for all $l, j, k$ where $l<i<j$.

4. Key Independence guarantees that a passive adversary who knows a proper subset of group keys $\hat{K} \subset \mathcal{K}$ cannot discover any other group key $\bar{K} \in(\mathcal{K}-\hat{K})$.

The relationship among the properties is intuitive. Either of Backward or Forward Secrecy subsumes Group Key Secrecy and Key Independence subsumes the rest. Also, the combination of Backward and Forward Secrecy forms Key Independence.

Our definition of group key secrecy allows partial leakage of information. Therefore, it would be more desirable to guarantee that any bit of the group key is unpredictable. For this reason, we prove a decisional version of group key secrecy in Appendix A. In other words, decisional version of group key secrecy guarantees that it is computationally infeasible for a passive adversary to distinguish any group key $K_{i}$ from random number.

Our definitions of Backward and Forward Secrecy are stronger than those typically found in the literature. The two are often defined (respectively) as [34,27]:

- Previously used group keys must not be discovered by new group members.

- New keys must remain out of reach of former group members.

The difference is that the adversary here is assumed to be a current or a former group member. Our definition additionally includes the cases of inadvertently leaked or otherwise compromised group keys. We refer to the above as Weak Forward Secrecy and Weak Backward Secrecy, respectively.

In this paper we do not assume key authentication as part of the group key management protocols. All communication channels are public but authentic. The latter means that all messages are digitally signed by the sender using some sufficiently strong public key signature method such as DSA or RSA.

In this paper we do not consider (implicit or explicit) key authentication as part of the group key management protocols. All communication channels are public but authentic. This means, as discussed later in the paper, that all messages are digitally signed by the sender using a sufficiently strong public key signature method, such as DSA or RSA. Consequently, our security model is different from the recent research $[12,13]$ that does not assume authentic channel. Furthermore, each message includes: the protocol identifier (TGDH), the event type identifier (i.e., JOIN, LEAVE, etc.), the protocol sequence number (or key epoch) and the sender's timestamp. All receivers are required to verify signatures on all received messages and check the aforementioned fields. Since no long-term secrets or other keys are used for encryption, we are not concerned with Perfect Forward Secrecy (PFS) since it is achieved trivially. 


\section{Notation and Definitions}

We use the following notation:

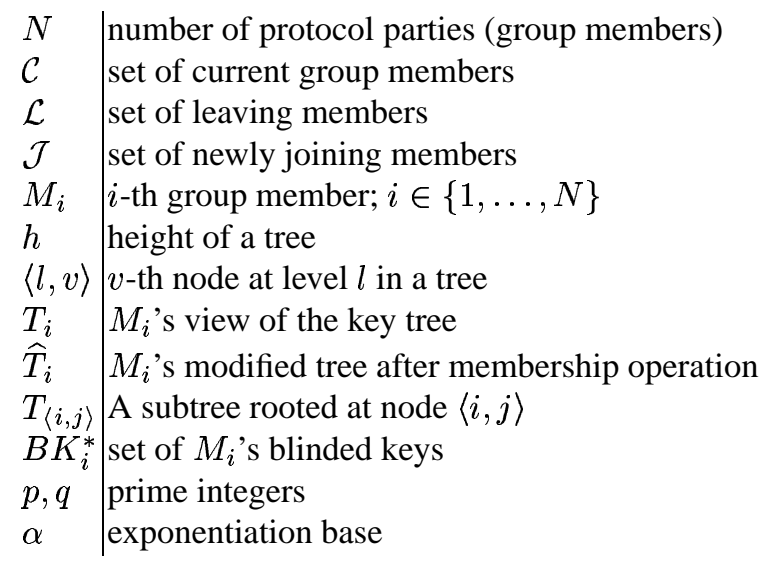

Key trees have been suggested in the past for centralized group key distribution systems. The seminal work of Wallner et al. [36] is the earliest such proposal. One of the main features of our work is the use of key trees in fully distributed contributory key agreement. Figure 1 shows an example of a key tree. The root is located at level 0 and the lowest leaves are at level $h$. Since we use binary trees, ${ }^{2}$ every node is either a leaf or a parent of two nodes. The nodes are denoted $\langle l, v\rangle$, where $0 \leq v \leq 2^{l}-1$ since each level $l$ hosts at most $2^{l}$ nodes. ${ }^{3}$ Each node $\langle l, v\rangle$ is associated with the key $K_{\langle l, v\rangle}$ and the blinded key (bkey) $B K_{\langle l, v\rangle}=f\left(K_{\langle l, v\rangle}\right)$ where the function $f()$ is modular exponentiation in prime order groups, i.e., $f(k)=\alpha^{k} \bmod p$ (analogous to the Diffie-Hellman protocol). Assuming a leaf node $\langle l, v\rangle$ hosts the member $M_{i}$, the node $\langle l, v\rangle$ has $M_{i}$ 's session random key $K_{\langle l, v\rangle}$. Furthermore, the member $M_{i}$ at node $\langle l, v\rangle$ knows every key along the path from $\langle l, v\rangle$ to $\langle 0,0\rangle$, referred to as the key-path and denoted $K E Y_{i}^{*}$. In Figure 1, if a member $M_{2}$ owns the tree $T_{2}$, then $M_{2}$ knows every key $\left\{K_{\langle 3,1\rangle}, K_{\langle 2,0\rangle}, K_{\langle 1,0\rangle}, K_{\langle 0,0\rangle}\right\}$ in $K E Y_{2}^{*}=\{\langle 3,1\rangle,\langle 2,0\rangle,\langle 1,0\rangle,\langle 0,0\rangle\}$ and every bkey $B K_{2}^{*}=\left\{B K_{\langle 0,0\rangle}, B K_{\langle 1,0\rangle}, \ldots, B K_{\langle 3,7\rangle}\right\}$ on $T_{2}$. Every key $K_{\langle l, v\rangle}$ is computed recursively as follows:

$$
\begin{aligned}
K_{\langle l, v\rangle} & =\left(B K_{\langle l+1,2 v+1\rangle}\right)^{K_{\langle l+1,2 v\rangle}} \bmod p \\
& =\left(B K_{\langle l+1,2 v\rangle}\right)^{K_{\langle l+1,2 v+1\rangle}} \bmod p \\
& =\alpha^{K_{\langle l+1,2 v\rangle} K_{\langle l+1,2 v+1\rangle}} \bmod p \\
& =f\left(K_{\langle l+1,2 v\rangle} K_{\langle l+1,2 v+1\rangle}\right)
\end{aligned}
$$

Computing a key at $\langle l, v\rangle$ requires the knowledge of the key of one of the two child nodes and the bkey of the other child node. $K_{\langle 0,0\rangle}$ at the root node is the group secret shared by all members. We stress, once again, that this value is never used as a cryptographic key for the purpose of encryption, authentication or integrity. Instead, such special-purpose sub-keys are derived from the group secret, e.g., by setting $K_{\text {group }}=h_{p}\left(K_{\langle 0,0\rangle}\right)$ where $h_{p}$ is a cryptographically strong hash function uniquely indexed with the purpose idenitifer $p$, e.g., encryption.

For example, in Figure $1, M_{2}$ can compute $K_{\langle 2,0\rangle}, K_{\langle 1,0\rangle}$ and $K_{\langle 0,0\rangle}$ using $B K_{\langle 3,0\rangle}, B K_{\langle 2,1\rangle}, B K_{\langle 1,1\rangle}$, and $K_{\langle 3,1\rangle}$. The final group key $K_{\langle 0,0\rangle}$ is:

$$
K_{\langle 0,0\rangle}=\alpha^{\left(\alpha^{r_{3}\left(\alpha^{\left.r_{1} r_{2}\right)}\right)}\right)\left(\alpha^{r_{4}\left(\alpha^{r_{5}} r^{6}\right)}\right)}
$$

To simplify subsequent protocol description, we introduce the term co-path, denoted as $C O_{i}^{*}$, which is the set of siblings of each node in the key-path of member $M_{i}$. For example, the co-path $C O_{2}^{*}$ of member $M_{2}$ in Figure 1 is the set of nodes $\{\langle 3,0\rangle,\langle 2,1\rangle,\langle 1,1\rangle\}$. Consequently, every member $M_{i}$ at leaf node $\langle l, v\rangle$ can derive the group secret $K_{\langle 0,0\rangle}$ from all bkeys on the co-path $C O_{i}^{*}$ and its session random $K_{\langle l, v\rangle}$.

\footnotetext{
${ }^{2}$ Note that the tree needs to be binary, since our protocol uses the two-party Diffie-Hellman key exchange to derive a node key from the contribution of the two children.

${ }^{3}$ Even though the key tree is not balanced, we assume a perfectly balanced tree for node numbering. Thus, a node's $\langle l, v\rangle$ left and right children have indexes $\langle l+1,2 v\rangle$ and $\langle l+1,2 v+1\rangle$, respectively.
} 


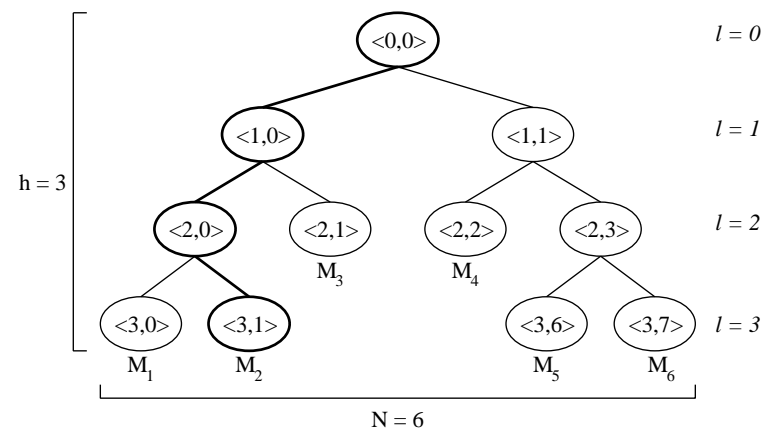

Fig. 1. Notation of a key tree

\section{TGDH Protocols}

In this section we introduce the four basic protocols that form the TGDH protocol suite: join, leave, merge, and partition. All protocols share a common framework with the following notable features:

- Each group member contributes an equal share to the group key. The key is computed as a function of all current group members' shares.

- Each share is secret (private to each group member) and is never revealed.

- As the group grows, new members' shares are factored into the group key and, upon each new member's joining, one of the old members changes its share.

- As the group shrinks, departing members' shares are removed from the new group key, and at least one remaining member changes its key share ${ }^{4}$.

- All protocol messages are signed, timestamped, sequence-numbered and type-identified by the sender; as discussed at the end of Section 3. (We use RSA for message signing since the number of receivers is greater than the number of senders. )

After every membership change, all remaining members independently update the key tree structure. Since we assume that the underlying communication system provides view synchrony (see Section 2), all members who correctly execute the protocol, recompute identical key trees after any membership event. The following is the minimal requirement for computing the group key:

Proposition 2. A group key can be computed from any member's secret share (i.e., any leaf value) and all bkeys on the co-path to the root.

It is easy to see that knowledge of its own secret share and all sibling bkeys on the path to the root enables a member to compute all intermediate keys on its key-path, including the root group key. This is similar to other treebased schemes $[38,36]$ where each member is required to know all keys on the path from itself (leaf) to the root. Although not strictly necessary for computing group key, our protocol also requires each member to know all bkeys in the entire key tree. As will be seen below, this makes the handling of future membership changes more efficient and robust.

As part of the protocol, a group member can take on a special sponsor role which involves computing intermediate keys and broadcasting to the group. Each broadcasted message contains the sender's view of the key tree which contains each bkey known to the sender. (We stress that intermediate keys are never broadcasted!) Any member in the group can unilaterally take on this responsibility, depending on the type of membership event. In some cases, such as a partition event, multiple sponsors might be involved.

\footnotetext{
${ }^{4}$ This prevents the group from reusing old keys. For example, if a member joins and immediately leaves, the group key would be the same before the join and after the leave. Although, in practice, this is not always a problem and might even be a desirable feature, we choose to err on the side of caution and change the key. In more concrete terms, changing the key upon all membership changes preserves key independence [34,7].
} 
In case of an additive change (join or merge), all group members identify a unique sponsor. This sponsor is responsible for updating its secret key share, computing affected $[k e y, b k e y]$ pairs and broadcasting all bkeys of the new tree to the rest of the group. The common criteria for sponsor selection is determined by the tree maintenance strategy described in Section 5.6. We emphasize, from the outset, that sponsor is not a privileged entity: its only task is the updating and broadcasting of tree information to the group.

In response to a subtractive membership change (leave or partition), all members update the tree in the same manner. Since the case of partition subsumes the case of a single leave, we discuss it in more detail. Group partition results in a smaller tree since some leaf nodes disappear. As a result, some subtrees acquire new siblings; therefore, new intermediate keys and bkeys must be computed through a Diffie-Hellman exchange between the new siblings sub-trees. The computation proceeds in a bottom-up fashion with each member computing keys and bkeys until either: 1) it blocks due to a dependency on a new sibling bkey that it does not yet know, or 2) it computes the new root (group) key. If a member blocks without computing any new keys, it does nothing. Otherwise, it broadcasts its view of the key tree which includes the newly computed bkeys. This process is repeated at most $h$ times where $h$ is the height of the tree, i.e., until all remaining members compute the new group key.

\subsection{TGDH Membership Events}

As discussed in Section 2, a group key agreement method needs to provide key adjustment protocols to cope with membership changes. TGDH includes protocols in support of the following operations:

- Join: a new member is added to the group

- Leave: a member is removed from the group

- Merge: a group is merged with the current group

- Partition: a subset of members are split from the group

- Key refresh: the group key is updated

Before turning our attention to the actual protocols we stress that, while a comprehensive protocol suite must address all types of key adjustment operations, the general policy (or case-by-case decisions) regarding if and when to change a group key is the responsibility of the application and/or the group communication system.

The following sections $(5.2-5.5)$, present the four protocols. In each section, we assume that every member can unambiguously determine both the sponsors and the insertion location in the key tree (in case of an additive event). Later in Section 5.6, we will explain how this works. Note that the key refresh operation can be considered a special case of leave without any members actually leaving the group.

\subsection{Join Protocol}

We assume the group has $n$ members: $\left\{M_{1}, \ldots, M_{n}\right\}$. The new member $M_{n+1}$ initiates the protocol by broadcasting a join request message that contains its own bkey $B K_{\langle 0,0\rangle}$. This message is distinct from any JOIN messages generated by the underlying group communication system, although, in practice, the two might be combined for efficiency's sake.

Each current member receives this message and determines the insertion point in the tree. The insertion point is the shallowest rightmost node, where the join does not increase the height of the key tree. Otherwise, if the key tree is fully balanced, the new member joins to the root node. The sponsor is the rightmost leaf in the subtree rooted at the insertion node. Next, each member creates a new intermediate node and a new member node, and promotes the new intermediate node to be the parent of both the insertion node and the new member node. After updating the tree, all members, except the sponsor, block. The sponsor proceeds to update its share and compute the new group key; it can do this since it knows all necessary bkeys. Next, the sponsor broadcasts the new tree which contains all bkeys. All other members update their trees accordingly and compute the new group key (see Proposition 2).

It might appear wasteful to broadcast the entire tree to all members, since they already know most of the bkeys. However, since the sponsor needs to send a broadcast message to the group anyhow, it might as well include more information which is useful to the new member, thus saving one unicast message to the new member (which would have to contain the entire tree).

Figure 3 shows an example of member $M_{4}$ joining a group where the sponsor $\left(M_{3}\right)$ performs the following actions:

1. renames node $\langle 1,1\rangle$ to $\langle 2,2\rangle$ 
Step 1: The new member broadcasts request for join.

$$
M_{n+1} \quad \longrightarrow K_{\langle 0,0\rangle}=\alpha^{r} n+1 \longrightarrow \quad \mathcal{C}=\left\{M_{1}, \ldots, M_{n}\right\}
$$

Step 2: Every member

- update key tree by adding new member node and new intermediate node,

- removes all keys and bkeys from the leaf node related to the sponsor to the root node. The sponsor $M_{s}$ additionally

- generates new share and computes all [key,bkey] pairs on the key-path,

- broadcasts updated tree $\widehat{T}_{s}$ including only bkeys.

$\mathcal{C} \cup\left\{M_{n+1}\right\}=\left\{M_{1}, \ldots, M_{n+1}\right\} \quad \widehat{T}_{s}\left(B K_{s}^{*}\right) \quad M_{s}$

Step 3: Every member computes the group key using $\widehat{T}_{s}$.

Fig. 2. Join Protocol

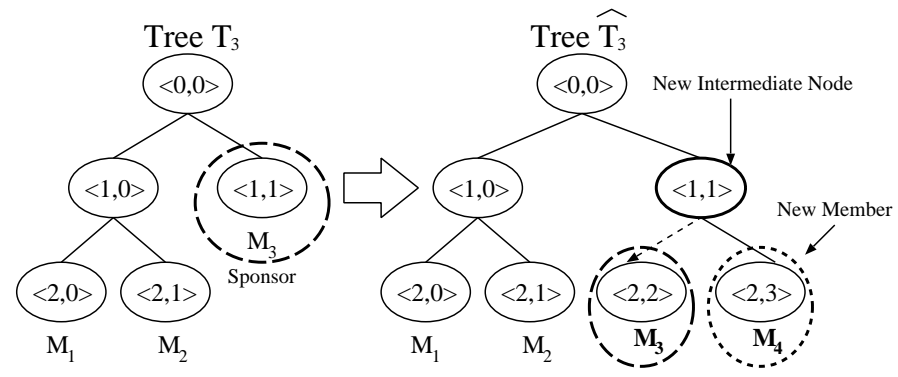

Fig. 3. Tree update: join 
2. generates a new intermediate node $\langle 1,1\rangle$ and a new member node $\langle 2,3\rangle$

3. promotes $\langle 1,1\rangle$ as the parent node of $\langle 2,2\rangle$ and $\langle 2,3\rangle$

Since all members know $B K_{\langle 2,3\rangle}$ and $B K_{\langle 1,0\rangle}, M_{3}$ can compute the new group key $K_{\langle 0,0\rangle}$. Every other member also performs step 1 and 2, but cannot compute the group key in the first round. Upon receiving the broadcasted bkeys, every member can compute the new group key.

Overall join operation requires 2 rounds and 3 messages to finish. Number of modular exponentiations ${ }^{5}$ depends on the joining point, which strands from 6 to $3 h-3$ where $h$ is the height of the resulting key tree. One of the example that costs most is when the new member joins to the leaf node of a balanced tree. In this case, the sponsor has to perform $2 h-2$ modular exponentiations (to compute $h-1$ keys and blinded keys each) and other members have to compute at most $h-1$ keys. Minimum number of mudular exponentiation is achieved when left or right subtree of the root node has single node like the example above. In this case, the sponsor needs to compute 4 modular exponentiations and other members need to compute at most 2 modular exponentiations.

\subsection{Leave Protocol}

Once again, we start with $n$ members and assume that member $M_{d}$ leaves the group. The sponsor in this case is the rightmost leaf node of the subtree rooted at the leaving member's sibling node. First off, as shown in Figure 4, each member updates its key tree by deleting the leaf node corresponding to $M_{d}$. The former sibling of $M_{d}$ is promoted to replace $M_{d}$ 's parent node. The sponsor generates a new key share, computes all [key,bkey] pairs on the key-path up to the root, and broadcasts the new set of bkeys. This allows all members to compute the new group key.

Looking at the setting in Figure 5, if member $M_{3}$ leaves the group, every remaining member deletes $\langle 1,1\rangle$ and $\langle 2,2\rangle$. After updating the tree, the sponsor $\left(M_{5}\right)$ picks a new share $K_{\langle 2,3\rangle}$, recomputes $K_{\langle 1,1\rangle}, K_{\langle 0,0\rangle}, B K_{\langle 2,3\rangle}$ and

$B K_{\langle 1,1\rangle}$, and broadcasts the updated tree $\widehat{T}_{5}$ with $B K_{5}^{*}$. Upon receiving the broadcast message, all members compute the group key. Note that $M_{3}$ cannot compute the group key, though it knows all the bkeys, because its share is no longer part of the group key.

One round and one message are required to complete a leave protocol. The number of modular exponentiation depends on the location of the leaving member and tree structure. Its upper bound is $3 h-3$ if all [key,bkey] pairs on the key-path of the deepest node need to be recomputed. When either left or right subtree has single node and it is the sponsor (i.e. for example, its sibling leaves the group), 3 modular exponentiations are required (two by the sponsor and one by all other members).

\subsection{Partition Protocol}

Assume that a network fault causes a partition of the $n$-member group. From the viewpoint of each remaining member, this event appears as a concurrent leave of multiple members. The partition protocol is involves multiple rounds; it runs until all members compute the new group key.

In the first round, each remaining member updates its tree by deleting all partitioned members as well as their respective parent nodes and "compacting" the tree. The procedure is as follows:

All leaving nodes are sorted by depth order. Starting at the deepest level, each pair of leaving siblings is collapsed into its parent which is then marked as leaving. This node is re-inserted into the leaving nodes list. The above is repeated until all leaving nodes are processed, i.e., there are no more leaving nodes that can be collapsed.

The resulting tree has a number of leaving (leaf) nodes but every such node has a remaining sibling node. Now, for each leaving node we identify a sponsor using the same criteria as described in Section 5.3.

Each sponsor now computes keys and bkeys on the key-path as far up the tree as possible. Then, each sponsor broadcasts the set of new bkeys. Upon receiving a broadcast, each member checks whether the message contains new bkeys. This procedure iterates until all members obtain the group key. (Recall that a member can compute the group key if it has all the bkeys on its co-path.)

\footnotetext{
${ }^{5}$ Hereafter, we count number of mudular exponentiations that need to be computed in serial.
} 
Step 1: Every member

- updates key tree by by removing the leaving member node and relevant parent node,

- removes all keys and bkeys from the leaf node related to the sponsor to the root node. Sponsor $M_{i}$ additionally

- generates new share and computes all [key,bkey] pairs on the key-path,

- broadcasts updated tree $\widehat{T}_{s}$ including only bkeys.

$$
M_{s} \quad \widehat{T}_{s}\left(B K_{s}^{*}\right) \longrightarrow\left\{M_{1} . . M_{n}\right\}-\left\{M_{d}\right\}
$$

Step 2: Every member computes the group key using $\widehat{T}_{s}$.

Fig. 4. Leave Protocol

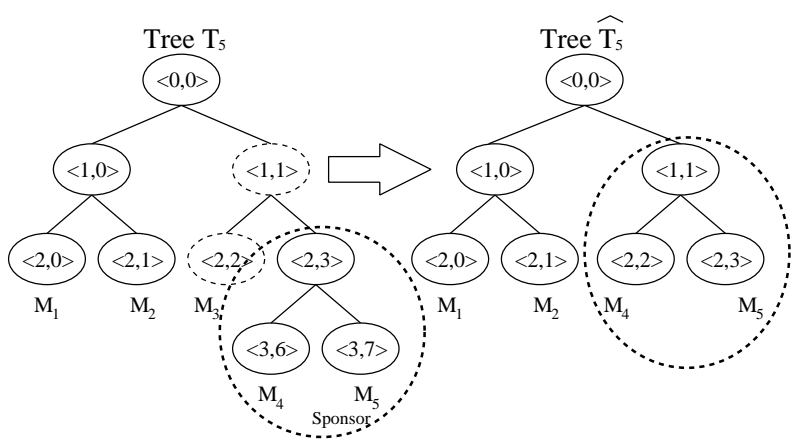

Fig. 5. Tree updating in leave operation

Step 1: Every member

- updates key tree by by removing all the leaving member nodes and their parent node,

- removes all keys and bkeys from the leaf node related to the sponsor to the root node.

- Each sponsor $M_{s_{t}}$

- If $M_{s_{t}}$ is the shallowest rightmost sponsor, generates new share,

- computes all [key, bkey] pairs on the key-path until it can proceed,

- broadcasts updated tree $\widehat{T}_{s_{t}}$ including only bkeys.

$$
M_{s_{t}} \stackrel{\widehat{T}_{s_{t}}\left(B K_{s_{t}}^{*}\right)}{\longrightarrow} \quad \mathcal{C}-\mathcal{L}
$$

Step 2 to $h$ (Until a sponsor $M_{s_{j}}$ computes the group key)

- Each sponsor $M_{s_{t}}$

- computes all [key, bkey] pairs on the key-path until it can proceed,

- broadcasts updated tree $\widehat{T}_{s_{t}}$ including only bkeys.

$$
M_{s_{t}} \stackrel{\widehat{T}_{s_{t}}\left(B K_{s_{t}}^{*}\right)}{\longrightarrow} \quad \mathcal{C}-\mathcal{L}
$$

Step $h+1$ : Every member computes the group key using $\widehat{T}_{s_{t}}$

Fig. 6. Partition Protocol 
To provide key independence, one of the remaining members needs to change its key share. For this reason, in the first round of the partition protocol, we require the shallowest rightmost sponsor to generate a new key share.

Figure 7 shows an example where all remaining members delete all nodes of leaving members and compute keys and bkeys in the first round. In the figure on the right, any of $M_{2}$ or $M_{3}\left(M_{5}\right.$ or $\left.M_{6}\right)$ cannot compute the new group key, since they lack the bkey $B K_{\langle 1,1\rangle}\left(B K_{\langle 1,0\rangle}\right)$, respectively. However, $M_{3}$ broadcasts $B K_{\langle 1,0\rangle}$ in the first round, and $M_{6}$ can thus compute the group key. Finally, every member knows all bkeys and can compute the group key. As discussed above, before computing $K_{\langle 1,1\rangle}, M_{6}$ changes its share $K_{\langle 2,3\rangle}$.

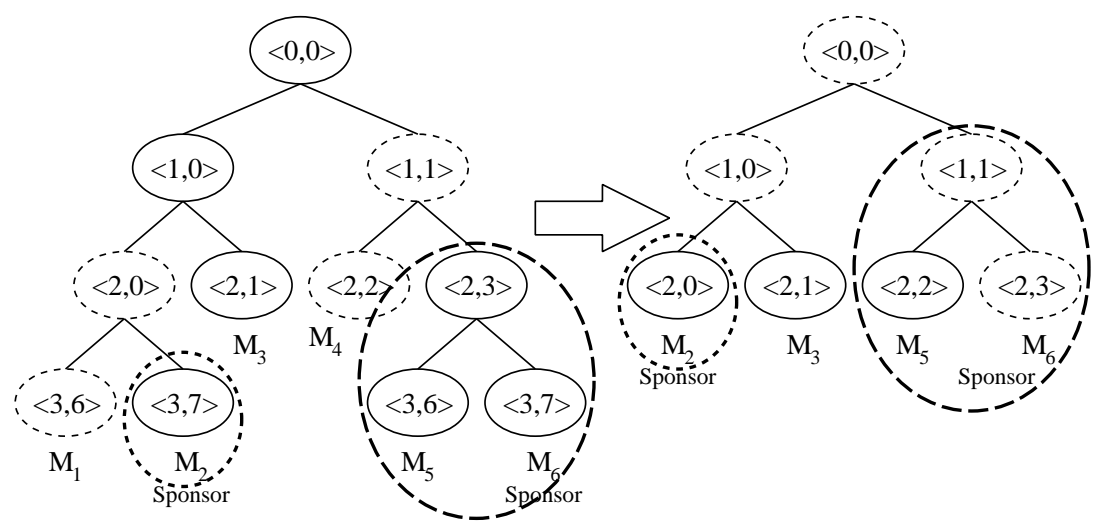

Fig. 7. Tree updating in partition operation

Note that, if some member $M_{i}$ computes the new group key in round $h^{\prime}$, then all other members can compute the group key, at the latest, in round $h^{\prime}+1$, since $M_{i}$ 's broadcast message contains all bkeys in the key tree. Hence, each member can detect the completion of the partition protocol independently.

The minimum and maximum number of modular exponentions for partition protocol are same as those of leave protocol. (The reason is obvious. Examples in leave protocol can be also applied here.) However, the communication costs are different. Two factors that affect the number of round are the number of leaving members and the resulting tree height. When $p$ members are partitioned from a group of $n$ members, each remaining member updates its tree by deleting all partitioned members as well as their respective parent nodes in the first round. Now, each key tree has at most $p$ paths with empty bkeys. The expected number of paths with empty keys is $p / 2$. Filling up these bkeys requires at most $\min \left(\left\lceil\log _{2} p\right\rceil+1, h\right)$ rounds, since 1$)$ every sponsor in each subsequent rounds computes bkeys as far up the tree as possible, and 2) the number of rounds never exceeds the tree height. Similarly, number of messages is $\min \left(2 p,\left\lceil\frac{n}{2}\right\rceil\right)$ in the worst case.

\subsection{Merge Protocol}

As discussed in Section 2, network faults can partition a group into several subgroups. After the network faults heal, subgroups may need to be merged back into a single group. We now describe the merge protocol for $k$ merging groups.

In the first round of the merge protocol, each sponsor (the rightmost member of each group) broadcasts its tree with all bkeys to all other groups after updating the secret share of the sponsor and relevant [key,bkey] pairs up to the root node. Upon receiving these messages, all members can uniquely and independently determine how to merge those $k$ trees by tree management policy described in 5.6.

Next, each sponsor computes all $[k e y, b k e y]$ pairs on the key-path until it either reaches the root or encounters a dependency. ${ }^{6}$ It then broadcasts its view of the tree to the group. All members update their tree views with the new information. If the broadcasting sponsor computed the root key, then, upon receiving the broadcast, all other members can compute the root key as well. In a more general case, a broadcast unblocks exactly one locked sponsor who can now compute further $[k e y, b k e y]$ pairs. This process is incremental, similar to the partition protocol. Finally, some sponsor will compute the new root key and will broadcast the key tree. Now, all members can compute the group key.

\footnotetext{
${ }^{6}$ If a sponsor cannot compute a new intermediate key, it does not broadcast but simply blocks.
} 


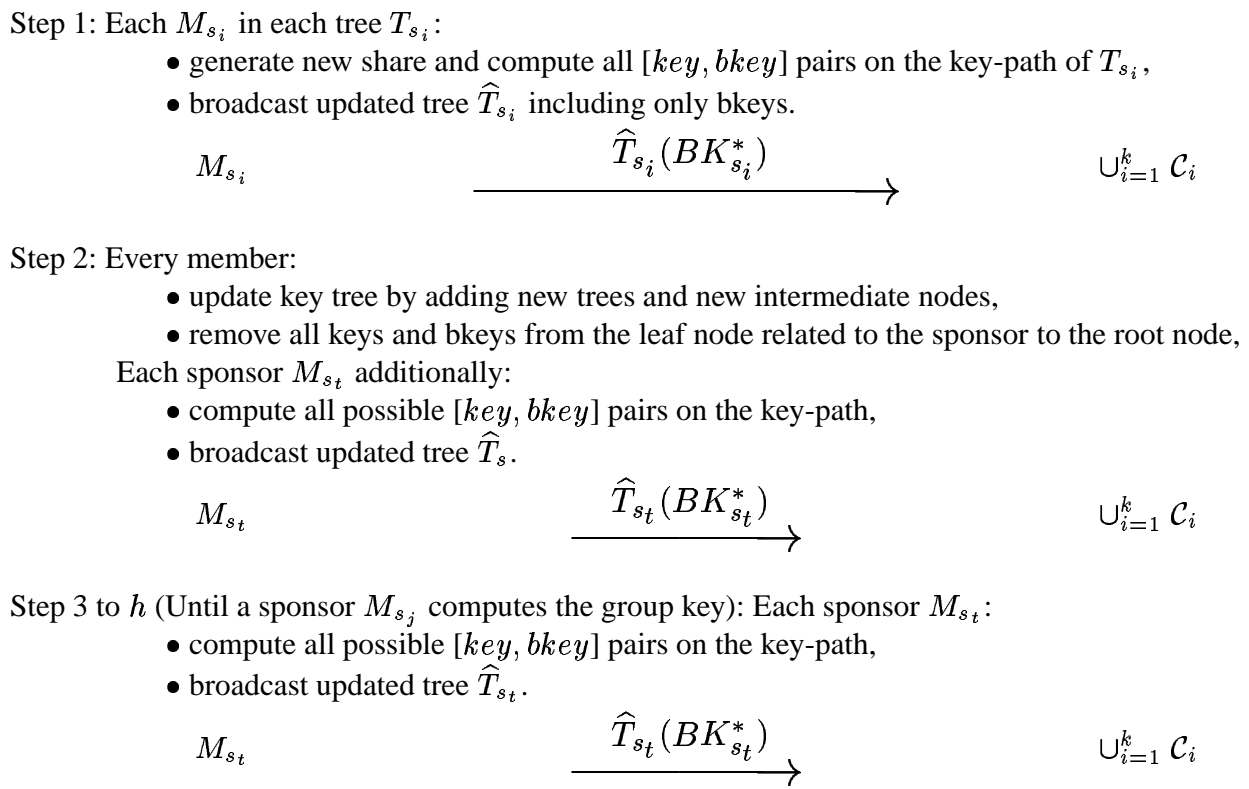

Fig. 8. Merge Protocol

The communication overhead of the merge protocol may appear high. However, this is not the case. Let us assume $k$ merging groups. In the first round, a sponsor in each group broadcasts its key tree after updating its session random. Upon receiving these broadcast messages, every member rebuilds a key tree which has some missing bkeys. At most $k$ paths will have missing bkeys. Propagating these bkeys requires at $\operatorname{most}\left\lceil\log _{2} k\right\rceil$ rounds, since each sponsor (in each subsequent round) computes bkeys as far as it can. Therefore, a merge of $k$ groups takes at most $\left\lceil\log _{2} k\right\rceil+1$ rounds. The maximum number of messages is $2 k$. The number of modular exponentions for the worst and the best case is same as that of join protocol, since rebuilding the whole key tree requires same amount of serial number of modular exponentions as joining to the leaf node.

Figure 9 shows an example of two merging groups, where the sponsors $M_{2}$ and $M_{7}$ broadcast their trees $\left(T_{2}\right.$ and $T_{7}$ ). Upon receiving these broadcast messages, every member checks whether it is the sponsor in the second round. Every member in both groups merges two trees, and then, $M_{2}$ (the sponsor in this example) updates the key tree, computes and broadcasts bkeys.

\subsection{Tree Management}

Modular exponentiation is the most computationally expensive operation in TGDH. The number of exponentiations for a membership event depends on the current tree structure and distance between the sponsor node and the root node. We can find good examples in join and leave operations. If the distance between the sponsor and the root node is $l$, the join and leave protocol requires $3 l$ modular exponentiations. Therefore, sponsor has to be chosen as near as possible to the root node.

Another important goal of tree management policy is to maintain the key tree as balanced as possible. If key tree is not well-balanced, future leave (partition) event may require many modular exponentiations.

In summary, our goal for the tree management policy is to:

- keep the key tree as balanced as possible, and

- minimize the number of modular exponentiations, and

- minimize the number of protocol rounds 


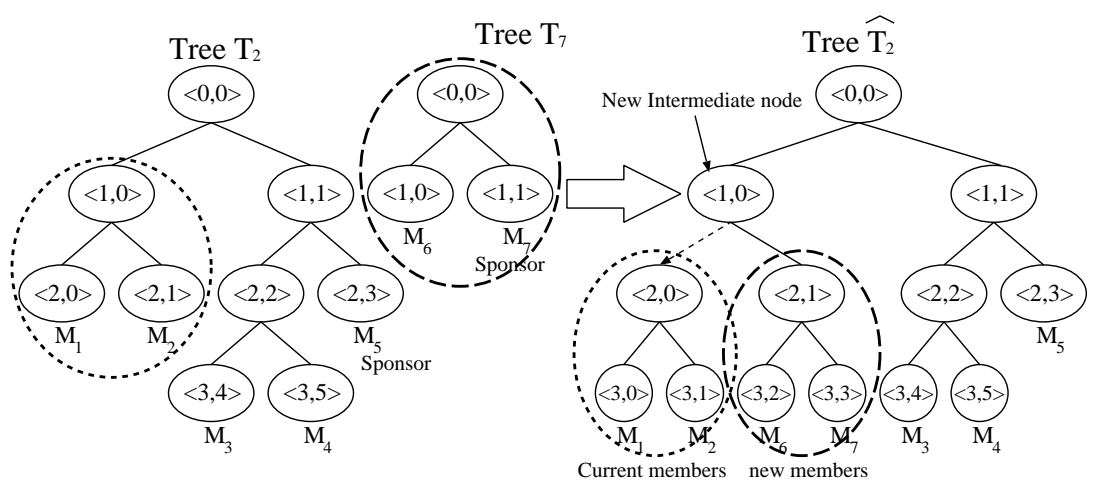

Fig. 9. Tree update in merge

5.6.1 Policy for Additive and Subtractive Events Our heuristic for keeping the key tree balanced is to choose the insertion node for a join or merge operation as the rightmost shallowest node. This usually does not increase the height. If we have to increase the height of the key tree, we simply join to the root. (See also Sections 5.2 and 5.5.) We do not employ any tree balancing scheme for the subtractive events or attempt to re-balance when the key tree becomes unbalanced.

In the rest of this section, we discuss our tree management policy for Merge (Join is a special case thereof). In particular, we focus on how each member independently, simultaneously, and unambiguously merges $k$ trees and selects an insertion point for each merge. Clearly, these properties (independency, concurrency, and consistency) are crucial to obtain a correct and efficient protocol.

Recall that we have $k$ merging trees. Each member invokes the merge_trees function $k-1$ times:

1. First, the trees are ordered from the highest $T_{1}$ to the lowest $T_{k}$. If multiple trees are of the same height, we list them in lexicographic order of the first member in each tree.

2. Let $\widehat{T}=T_{1}$.

3. For $i=2$ to $k, \widehat{T} \longleftarrow$ merge_trees $\left(\widehat{T}, T_{i}\right)$.

Since every member can order the merging trees independently and unambiguously, all members can agree on a key tree if the merge_trees algorithm guarantees uniqueness of the result. We now show how to merge two trees.

If two trees are of the same height, we join one tree to the root node (insertion point) of the other. To provide unambiguous ordering we lexicographically compare the identifiers of the respective sponsors. Otherwise, we join the shallower tree to the deeper tree. To locate the insertion point we first try find the rightmost shallowest node (not necessarily a leaf) where the join would not increase the overall tree height. If no such node exists (i.e., the tree height would increase anyway) the insertion point is the root node.

As an illustration, consider two trees $T_{h}$ and $T_{l}$, where the height of $T_{h}$ is greater than that of $T_{l}$. The merge_trees algorithm is as follows:

The first if statement in the while loop breaks when there is no join-able node in $T_{h}$; the trees will then be joined at the root node. Join-able means that we can merge two trees without increasing the height of $T_{h}$ by placing a subtree rooted at the join-able node as the left child of itself, and putting $T_{l}$ as the right child. We can see that merge_trees algorithm fulfills the goal for the tree management policy described above.

5.6.2 Sponsor Selection Summary Sponsor selection in TGDH takes place in each protocol round. (Recall that TGDH is a multi-round protocol.) As mentioned earlier, uniqueness, consistency and independence of this process are crucial for protocol correctness. Sponsor selection is performed as follows.

We already mentioned the behavior of the sponsor in two situations:

1. Additive event: member associated with the rightmost shallowest leaf node of each key tree becomes the sponsor.

2. Subtractive event: member associated with the rightmost shallowest leaf node rooted at the sibling node of each leaving member. In case of partition, there may be multiple sponsors. 


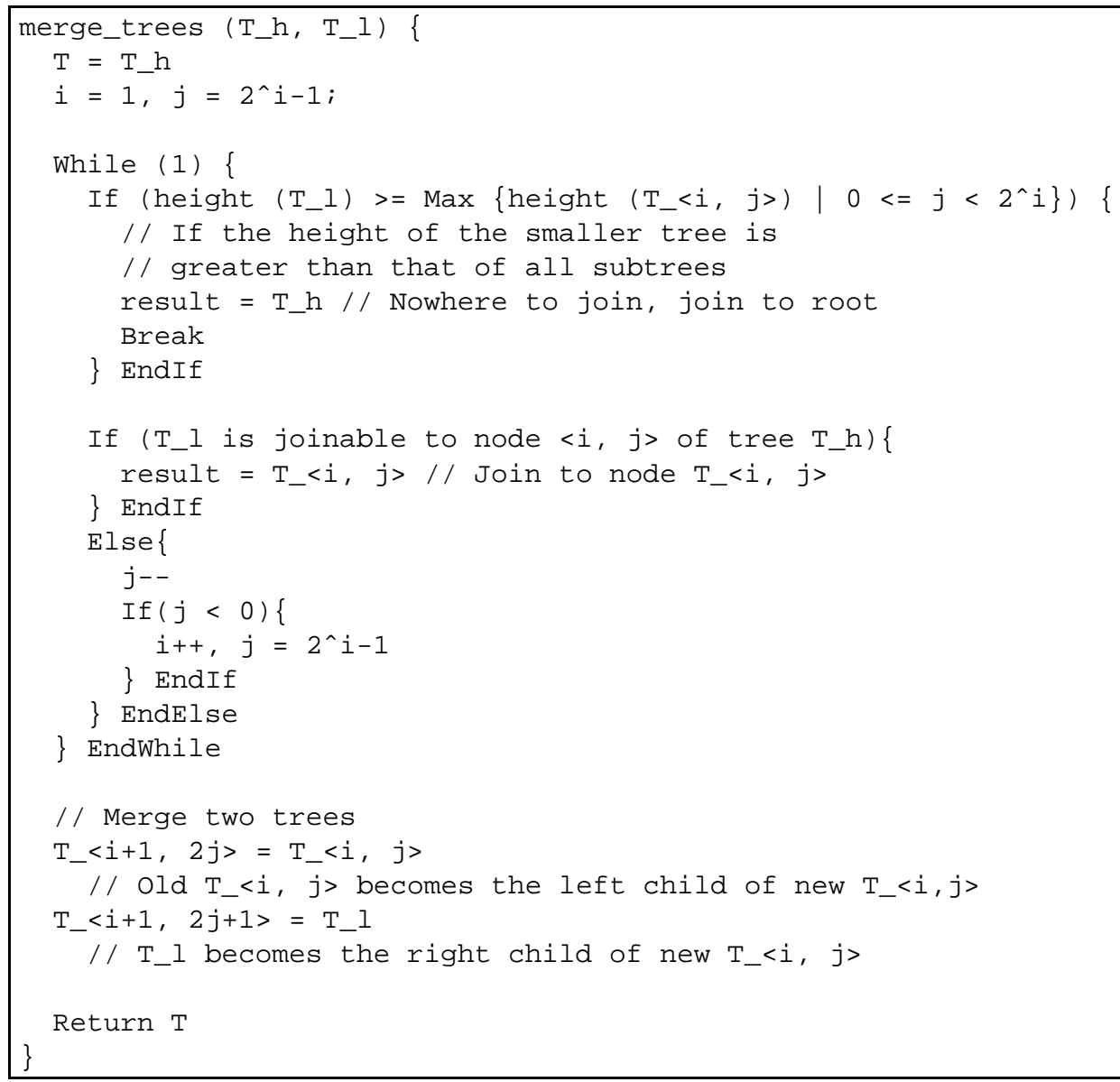


The above only covers the initial protocol round. In subsequent rounds, a sponsor is always the rightmost shallowest leaf rooted at the node which lacks a current bkey.

To summarize, the role of a sponsor is three-fold: 1) refresh its key share $\left.{ }^{7}, 2\right)$ compute all $[k e y, b k e y]$ pairs as far on the key-path as possible, and 3) broadcast the updated key tree to all current group members.

\section{Practical Considerations}

In this section, we describe the TGDH implementation issues and then discuss self-stabilization and self-clustering properties.

\subsection{Implementation Architecture}

TREE_API is a group key agreement API that implements the cryptographic primitives of TGDH. It contains the following three function calls:

- tree_new_user: called by any new member to generate its context.

- tree_merge_req: called by every group member when a join/merge occurs. It identifies the sponsor unambiguously (as described in Section 5.6). It then removes all [key, bkey] pairs on its key-path. If the caller is a sponsor, generates new secret share and computes all keys and bkeys on its key-path. This function returns an output token, which is then broadcast to the whole group.

- tree_cascade: invoked by every member when a subtractive event happens or when all members try to compute the group key collaboratively. In the former case, this function removes all leaving members and their parents as described in Section 5.3. If the caller is a sponsor, it also tries to compute [key,bkey] pairs on the sponsor's key-path. In the latter case, this function is called repeatedly until the group key is computed.

The underlying communication system is assumed to deal with group communication and network events such as merges, partitions, failures and other abnormalities. ${ }^{8}$ We use OpenSSL 0.9.6 [26] as the underlying cryptographic library.

In the following Sections (6.2 and 6.3), we show that tree_cascade provides robustness against cascaded network events. Since TREE_API does not provide its own communication facility, the robustness of the API was tested by simulating random events on a single machine running all group members.

\subsection{Protocol Unification}

Although described separately in the preceding sections, the four TGDH operations (join, leave, merge and partition) actually represent different strands of a single protocol. We justify this claim with an informal argument below.

Obviously, join and leave are special cases of merge and partition, respectively. We observe that merge and partition can be collapsed into a single protocol, since, in either case, the key tree changes and remaining group members lack some number of bkeys. This prevents them from computing the new root key. In a partition, the remaining members (in any surviving group fragment) reconstruct the tree where some bkeys are missing. In case of a merge of two groups, let us suppose that a taller (deeper) tree $\mathcal{A}$ is merged with a shorter (shallower) tree $\mathcal{B}$. Similar to a partition, all members formerly in $\mathcal{A}$ construct the new tree where some bkeys - those in $\mathcal{B}$ - are missing. (This view is symmetric since the members in $\mathcal{B}$ see the same tree but with missing bkeys in the subtree $\mathcal{A}$.)

We now established that both partition and merge initially result in a new key tree with a number of missing bkeys. In the first round of merge protocol, sponsor in each group broadcasts the key tree after updating its session random. Upon receiving this broadcast message, every member rebuilds a key tree which has some missing bkeys. Filling up this bkeys takes at most $\log _{2} k$ rounds. A partition is very similar except the first broadcast message of merge.

The apparent similarity between partition and merge allows us to collapse the protocols stemming from all membership events into a single unified protocol. Figure 10 shows the pseudocode. The incentive for doing this is threefold. First, unification allows us to simplify the implementation and minimize its size. Second, the overall security and correctness are easier to demonstrate with a single protocol. Third, we can now claim that (with a slight modification) TGDH is self-stabilizing and fault-tolerant as discussed below.

\footnotetext{
${ }^{7}$ In a join, the new member simply generates its first share.

${ }^{8}$ Currently, TGDH is integrated with Spread [4] group communication system.
} 


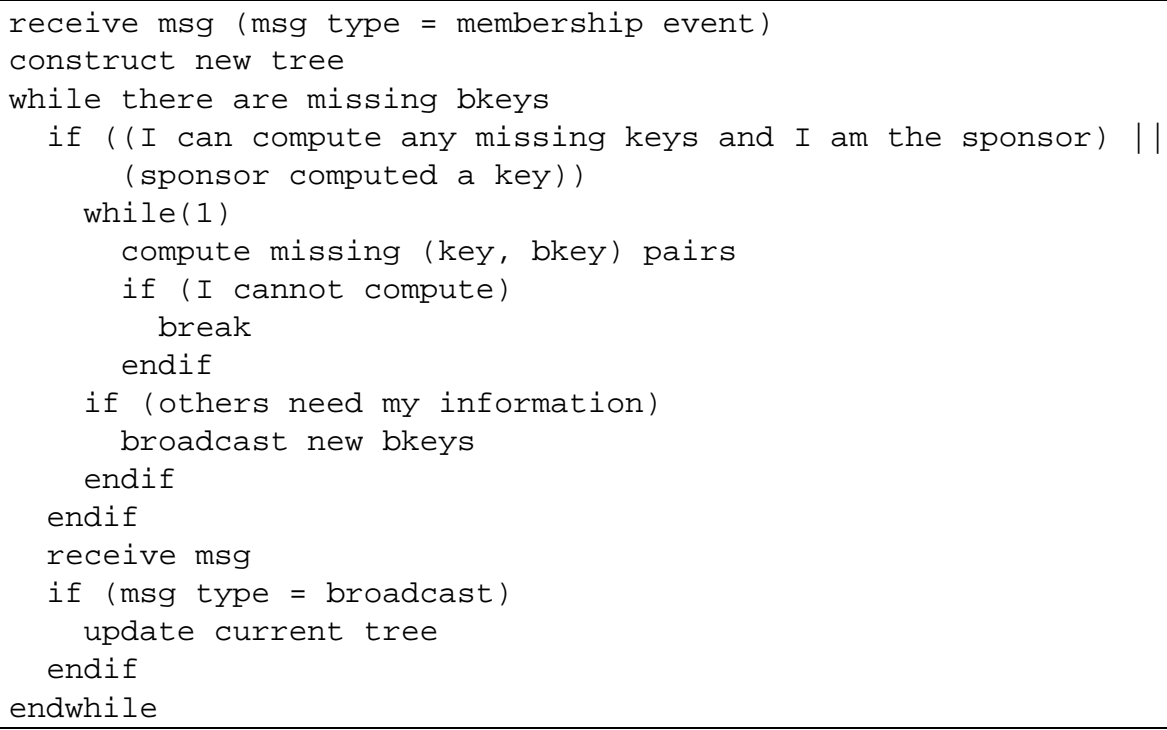

Fig. 10. Unified protocol pseudocode

\subsection{Cascaded Events}

Since network disruptions are random and unpredictable, it is natural to consider the possibility of so-called cascaded membership events. (In fact, cascaded events and their impact on group and multi-round protocols are often considered in group communication literature, but, alas, not often enough in the security literature.) A cascaded event occurs, in a simplest form, when one membership change occurs while another is being handled. Here event means any of: join, leave, partition, merge or any combination thereof. For example, a partition can occur while a prior partition is being dealt with, resulting in a cascade of size two. In principle, cascaded events of arbitrary size can occur if the underlying network is highly volatile.

We claim that the TGDH partition protocol is self-stabilizing, i.e., robust against cascaded network events. This property is notable and rare as most multi-round cryptographic protocols are not geared towards handling of such events. In general, self-stabilization is a very desirable feature since lack thereof requires extensive and complicated protocol "coating" to either: 1) shield the protocol from cascaded events, or 2) harden it sufficiently to make the protocol robust with respect to cascaded events (essentially, by making it re-entrant).

The high-level pseudocode for the self-stabilizing protocol is shown in Figure 11. The changes from Figure 10 are minimal (lines 18 - 19 are added).

Instead of providing a formal proof of self-stabilization we demonstrate it with an example. Figure 12 shows an example of a cascaded partition event. The first part of the figure depicts a partition of $M_{1}, M_{4}$, and $M_{7}$ from the prior group of ten members $\left\{M_{1}, \ldots, M_{10}\right\}$. This partition normally requires two rounds to complete the key agreement. As described in Section 5.4, every member constructs the same tree after completing the initial round. The middle part shows the resulting tree. In it, all non-leaf nodes except $K_{\langle 2,3\rangle}$ must be recomputed as follows:

1. First, $M_{2}$ and $M_{3}$ both compute $K_{\langle 2,0\rangle}, M_{5}$ and $M_{6}$ compute $K_{\langle 2,1\rangle}$ while $M_{8}, M_{9}$ and $M_{10}$ compute $K_{\langle 1,1\rangle}$. All bkeys are broadcasted by each sponsor $M_{2}, M_{5}$ and $M_{8}$.

2. Then, as all broadcasts are received, $M_{2}, M_{3}, M_{5}$ and $M_{6}$ compute $K_{\langle 1,0\rangle}$ and $K_{\langle 0,0\rangle}$. The bkeys are broadcasted by the sponsor $M_{6}$.

3. Finally, all broadcasts are received and $M_{8}, M_{9}$ and $M_{10}$ compute $K_{\langle 0,0\rangle}$.

Suppose that, in the midst of handling the first partition, another partition (of $M_{3}$ and $M_{8}$ ) takes place. Note that, regardless of which round $(1,2,3)$ of the first partition is in progress, the departure of $M_{3}$ and $M_{8}$ does not affect the keys (and bkeys) in the subtrees formed by $M_{9}$ and $M_{10}$ as well as $M_{5}$ and $M_{6}$. All remaining members update the tree as shown in the rightmost part of Figure 12 . The bkey of $K_{\langle 1,0\rangle}$ is the only one missing in all members' view 


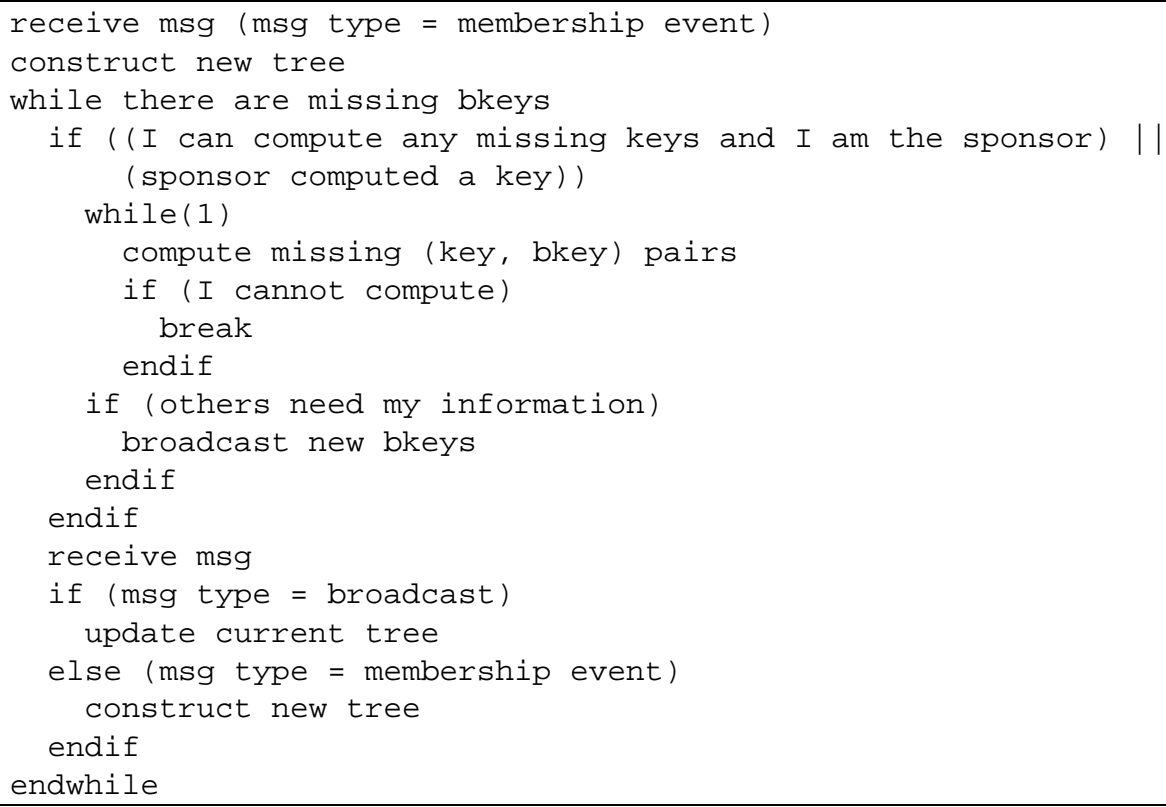

Fig. 11. Self-stabilizing protocol pseudocode

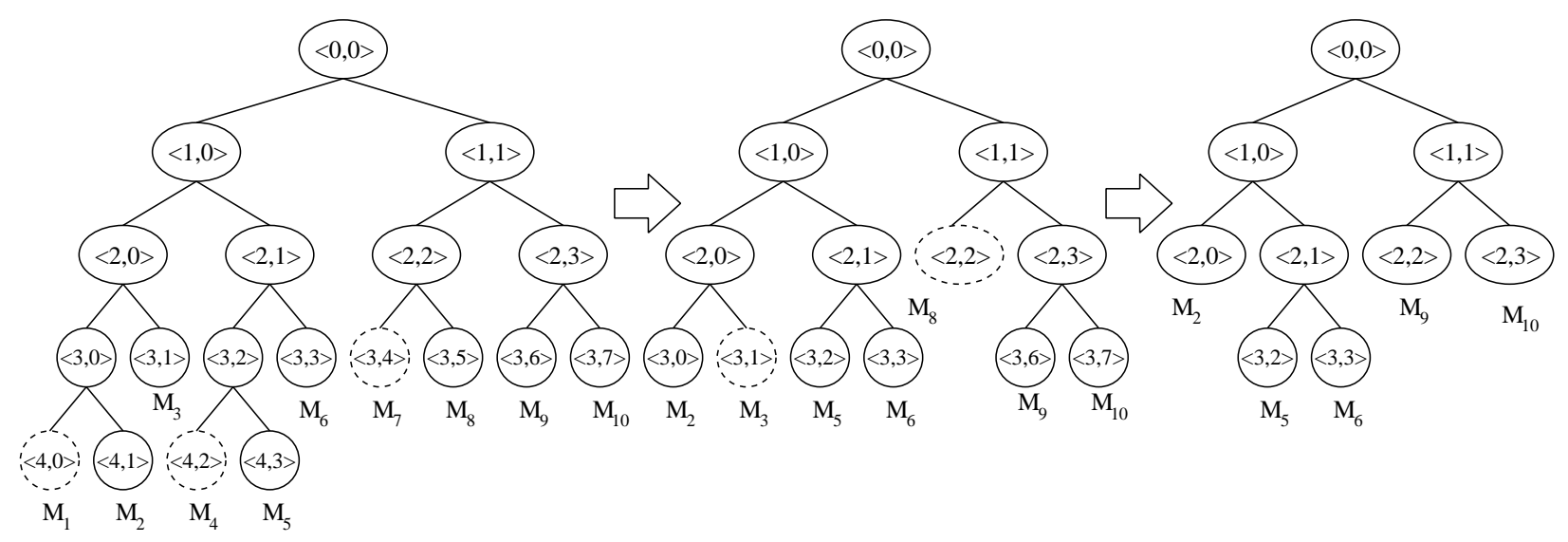

Fig. 12. An Example of Cascaded Partition 
of the tree. It is computed by $M_{2}, M_{5}$ and $M_{6}$ and broadcasted by $M_{6}$. When the broadcast is received, all members compute the root key.

The only remaining issue is whether a broadcast from the first partition can be received after the notification of the second (cascaded) partition. Here we rely on the underlying group communication system to guarantee that all membership events are delivered in sequence after all outstanding messages are delivered. In other words, if a message is sent in one membership view and membership changes while the message is not yet delivered, the membership change must be postponed until the message is delivered to the (surviving) subset of the original membership. This is essentially a restatement of View Synchrony (as discussed in Section 2).

\subsection{Self-Clustering}

The Internet as a whole provides sporadic and unstable connectivity, e.g., web users frequently experience disconnects and server failures. The instability can occur because of congestion, equipment failures or lossy links. It can also take place as a result of denial-of-service attacks, worms and viruses. It is often the case that an unstable network component (router or link) tends to have multiple failures. In other words, an isolated, "once-in-a-blue-moon" type of failure is uncommon. Repeated failures typically complicate protocol implementation. However, oddly enough, TGDH not only survives but also benefits from repeated failures.

Similar to other tree-based key management schemes (e.g., [36, 38, 24]) the key tree in TGDH is logical: group members are leaves in a tree and internal nodes are logical. The initial placement of members (as tree leaves) is not dependent on their relative physical location. Therefore, members physically close to each other might not be neighbors in a key tree. When a partition occurs, all members in the same physical group fragment form a new key tree and a new group. The partition protocol may cost as many as $\log n$ rounds. Then, when the partition heals, the previously separate groups are merged into a single key tree, however, they are still clustered along the lines of the partition. If another partition happens on the same link, the partitioned members are not scattered across the key tree any longer. Therefore, any subsequent partition on the same link will take only one round to complete. This property is especially important in high-delay wide area networks since clustering lowers the number of communication rounds as well as the number of modular exponentiations, in many cases.
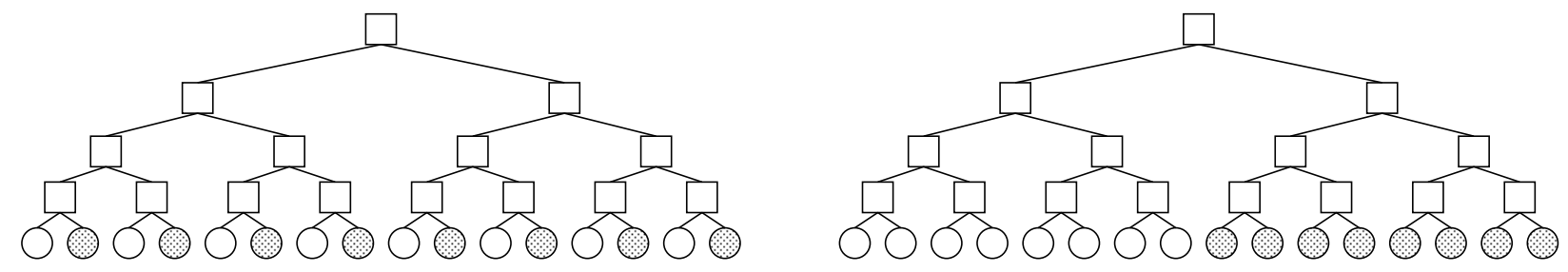

First Partitions on a weak link L

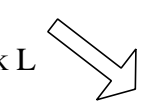

All merges

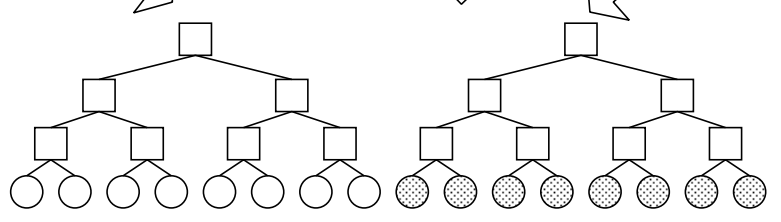

All other partitions on the weak link L

Fig. 13. An Extreme Example of Self-Clustering

Figure 13 shows an extreme example of self-clustering. Suppose that a group has sixteen members numbered $M_{1}$ through $M_{16}$ where white odd-numbered nodes are located in one physical cluster (e.g., a LAN) and shaded evennumbered nodes in another. The two partitions are connected via an unstable link $L$. If $L$ fails and a partition occurs, it takes three rounds to complete the partition protocol. It can be clearly seen that each group forms a cluster after the partition. When $L$ comes up and the partition heals (i.e., a merge occurs), two rounds are needed to complete the merge protocol. Subsequently, all partitions on link $L$ will require only one round and all merges - two rounds. 


\section{Performance Analysis}

\subsection{Complexity Analysis and Comparison}

We analyze the communication and computation costs for join, leave, merge and partition protocols. We focus on the number of rounds, the total number of messages, the serial number of exponentiations, the serial number of signature generations, and the serial number of signature verifications. Note that we use RSA signature for message authentication since RSA is particularly efficient in verification. The serial cost assumes parallelization within each protocol round and represents the greatest cost incurred by any participant in a given round. The total cost is the sum of all participants' costs in a given round.

We also compare our protocol to other contributory group key agreement schemes including GDH.3 [34], BD (Burmester-Desmedt) [14], and STR [22]. Although BD was originally designed to support only group formation, we modify the BD protocol to support dynamic membership operation. This modification is minimal.

Table 1 summarizes the communication and computation costs of four protocols. The numbers of current group members, merging members, merging groups, and leaving members are denoted as: $n, m, k$ and $p$, respectively.

The height of the key tree constructed by the TGDH protocol is $h$. The overhead of the TGDH protocol depends on the tree height, the balancedness of the key tree, the location of the joining tree, and the leaving nodes. In our analysis, we assume the worst case configuration and list the worst-case cost for TGDH.

Table 1. Communication and Computation Costs

\begin{tabular}{|c|c|c|c|c|c|c|}
\hline & \multicolumn{2}{|c|}{ Communication } & \multicolumn{3}{|c|}{ Computation } \\
\hline & & Rounds & Messages & Exponentiations & Signatures & Verifications \\
\hline \multirow{4}{*}{ GDH } & Join & 4 & $n+3$ & $n+3$ & 4 & $n+3$ \\
\hline & Leave & 1 & 1 & $n-1$ & 1 & 1 \\
\hline & Merge & $m+3$ & $n+2 m+1$ & $n+2 m+1$ & $m+3$ & $n+2 m+1$ \\
\hline & Partition & 1 & 1 & $n-p$ & 1 & 1 \\
\hline \multirow{4}{*}{ TGDH } & Join & 2 & 3 & $3 h-3$ & $\overline{2}$ & 3 \\
\hline & Leave & 1 & 1 & $3 h-3$ & 1 & 1 \\
\hline & \begin{tabular}{|l|} 
merge \\
\end{tabular} & $\left.\mid \log _{2} k\right\rceil+1$ & $2 k$ & $3 h-3$ & $\left\lceil\log _{2} k\right\rceil+1$ & $\left\lceil\log _{2} k\right\rceil$ \\
\hline & Partition & $\mid \min \left(\left\lceil\log _{2} p\right\rceil+1, h\right)$ & $\min \left(2 p,\left\lceil\frac{n}{2}\right\rceil\right)$ & $3 h-3$ & $\min \left(\left\lceil\log _{2} p\right\rceil+1, h\right)$ & $\min \left(2 p,\left\lceil\frac{n}{2}\right\rceil\right)$ \\
\hline \multirow{4}{*}{ STR } & Join & || 2 & 3 & 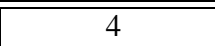 & 2 & 3 \\
\hline & Leave & 1 & 1 & $\frac{3 n}{2}+2$ & 1 & 1 \\
\hline & \begin{tabular}{|l|} 
Merge \\
\end{tabular} & 2 & $k+1$ & $3 m+1$ & 2 & 3 \\
\hline & Partition & 1 & 1 & $\frac{3 n}{2}+2$ & 1 & 1 \\
\hline \multirow{4}{*}{$\mathrm{BD}$} & Join & 2 & $2 n+2$ & 3 & 2 & $n+3$ \\
\hline & Leave & 2 & $2 n-2$ & 3 & 2 & $n+1$ \\
\hline & Merge & 2 & $2 n+2 m$ & 3 & 2 & $n+m+2$ \\
\hline & Partition & 2 & $2 n-2 p$ & 3 & 2 & $n-p+2$ \\
\hline
\end{tabular}

The BD protocol has a hidden cost that is not listed in Table 1: BD has $n-1$ modular exponentiations with a small exponent. Unfortunately, $n-1$ such exponentiations can be expensive when $n$ is large. For example, BD requires $O\left(n^{2}\right)$ 1024-bit modular multiplications, if modular exponentiation is implemented with the square-and-multiply algorithm. (OpenSSL uses Montgomery reduction and the sliding window algorithm to implement the modular exponentiation, which is faster than simple square-and-multiply algorithm. However, the former requires almost the same time as the latter for small exponents.) Because of this hidden cost, it is hard to compare the computational overhead of BD to the other protocols. Below, we compare the four protocols for each membership event.

Join: All protocols except GDH.3 require two communication rounds. In terms of communication, the most expensive protocol is BD which involves $n$ messages (all broadcast) in each round. Other protocols use a constant number of messages. GDH is the most expensive in terms of computation, requiring linear number of exponentiations. TGDH is comparatively efficient, scaling logarithmically in the number of exponentiations. STR has a constant number of modular exponentiations. BD requires the least exponentiations, but has the hidden cost. 
Leave: BD is the most expensive protocol in terms of communication. The cost order among others is determined strictly by the computation cost, since they all have the same communication cost (one round consisting of one message). Therefore, TGDH is best for handling leave events. STR, and GDH scale linearly with the group size. BD has a hidden cost, which makes it hard to compare.

Merge: We first look at the communication cost. GDH scales linearly with the number of added members, while BD and STR are more efficient with a constant number of rounds. Although a merge in TGDH takes multiple rounds, it depends on the number of merging groups, which is usually small. Since BD and TGDH have $2 n$ and $2 k$ messages (at most) respectively, STR is the most communication-efficient for handling merge events. Examining computation requirements, BD has the lowest cost with only three exponentiations. TGDH scales logarithmically with the group size. It is more efficient than STR and GDH which scale linearly with both the group size and the number of new members.

Partition: Both GDH and STR protocols are bandwidth efficient: only one round consisting of one message. BD is less efficient with two rounds of $n$ messages each. Partition is the most expensive operation in TGDH requiring a number of rounds bounded by tree height. Computation-wise it is difficult to compare BD with other protocols because of its hidden cost. TGDH requires a logarithmic number of exponentiations. GDH and STR scale linearly with the group size.

\subsection{Experimental Results}

To compare the actual performance, we implemented the four protocols and compared their costs in this section. We simulated the total computation delay from the time when the membership event happens to the time when group key agreement finishes. Average delay has been measured, since all members do not finish group key agreement simultaneously.

7.2.1 Test Methodology To perform fair comparisons, we consider the followings:

- We use $p=1024$ and $q=160$ for all measurements. These values are known to be secure in the current technology [23].

- We use 1024-bit RSA signature with the fixed public exponent 3 for message authentication. All protocols have multiple signature verifications that need to be processed serially. No security risk is known for RSA signatures with small public exponents [11].

- For TGDH, we first generate a random tree by forcing a number of random partition/merge events. Since the cost of TGDH depends on the tree structure, it is fair to generate a random tree instead of a well-balanced or an imbalanced tree.

We use the following scenario to measure delay. For join and leave, the number of current group members is $n$. For partition and merge, $n$ varies among: 16, 32, 64, and 128.

Join We measure the computational delay for a member to join a group of $n$ members. (Left graph of Figure 14) In case of TGDH, we use a random tree as described above. The $x$-axis denotes the number of current group members, while the $y$-axis shows the computational delay in seconds.

Leave We measure the computational delay for a random member to leave a group of $n$ members. (Right graph of Figure 14) Note that the delay for GDH and BD does not depend on the location of the leaving member. However, the number of modular exponentiations for STR upon a leave event depends on the location of the leaving node. For TGDH, we pick a random member from the tree, and measure the average delay for the leave. The $x$-axis denotes the number of remaining group members and the $y$-axis is the computational delay in seconds.

Partition We measure the computational delay after a partition. If the number of current group members is $n$ and this group shrinks to group of size $k$, we measure the average delay for the remaining group members. For BD and GDH, the location of the leaving members does not matter. However, it is important in STR and TGDH. We, therefore, choose leaving members at random. In Figure 15, the $x$-axis denotes the number of remaining group members. 
Merge Merge is the trickiest algorithm to measure fairly. First, in BD and GDH, only the number of resulting members decides the total delay, independent of the number of merging groups. Second, the performance of STR merge depends on the size of the largest group (which decides the number of modular exponentiation), and the number of groups merging (which determines the number of signature verifications). Finally, the performance of TGDH merge depends upon the number of merging groups (which affects the number of signature generations and verifications), and the key tree structure. The number of current group members is not important for TGDH.

Since each protocol has different characteristics, we measured the merge costs as follows:

- The number of resulting group members is $16,32,64$ and 128 .

- We assume the maximum number of merging groups is five. In practice, merge of two groups is the most frequent event. However, we allow up to five groups since some group communication systems may allow (require) more than two groups to merge at one time.

- For TGDH and STR, values in the $x$-axis mean the number of current group members. The resulting group size is $16,32,64,128$, respectively. The values in the $y$-axis are the average computational delays for a member in the current group after a merge of $2-5$ groups.
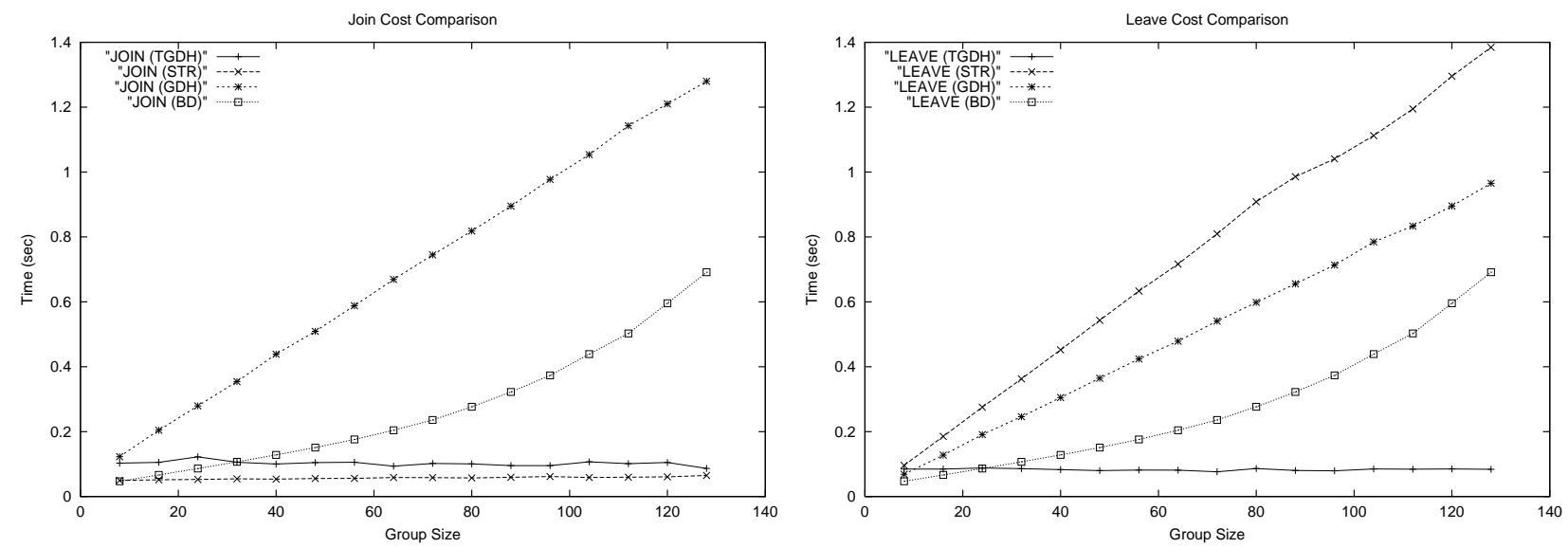

Fig. 14. Join and Leave Cost Comparison: $(x, y)=$ (number of remaining group members after JOIN/LEAVE, computational overhead in seconds)

7.2.2 Join Results. The left graph of Figure 14 depicts measurement for join. As expected, STR has the smallest delay. A surprising result comes from the TGDH for a random tree: the difference between TGDH and STR is small. In case of a random tree, the joining node is located close to the root node. GDH is the worst performer due to many modular exponentiations. BD also shows interesting results. Though it has constant number of exponentiations, the hidden cost evidently plays an important role.

7.2.3 Leave Results. As expected, STR is the worst performer. Note that the worst case (when a lowest member leaves the group) cost for STR is almost twice as much as the current average value. Performance of TGDH looks best overall, while BD performs very well when the number of group members is less than 25 . Leave cost in BD is almost the same as join cost, since the protocol needs to restart whenever a new membership event happens.

7.2.4 Partition Results. Figure 15 shows partition cost when the number of current group members is 16, 32, 64 , and 128 respectively. As expected from the conceptual results, STR has the worst performance due to many modular exponentiations. TGDH shows an interesting graph: it increases until $40 \%$ of the group members leave the group, 
and decreases afterwards. This is because 1) as the number of leaving members increases, the number of modular exponentiations decreases, 2) when many members leave the group, the resulting group has many empty bkeys spread over the tree, and, hence, requires more messages. The cost of BD and GDH decreases almost linearly, because it depends on the number of resulting group members.

As described in Section 6.4, the cost of partition for TGDH can be improved when the group experiences repeated network partition on the same link.
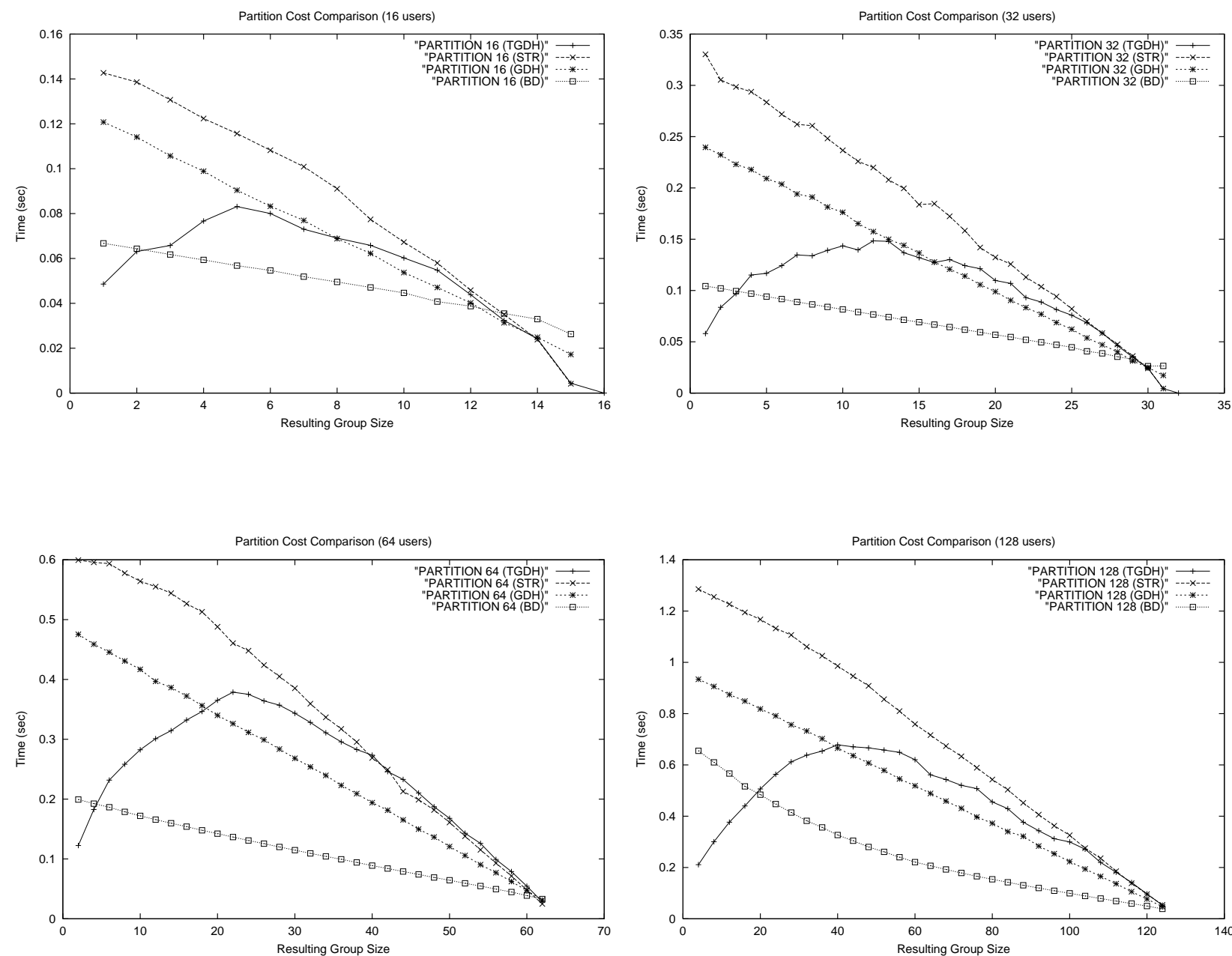

Fig. 15. Partition Cost Comparison: $(x, y)=$ (number of remaining group members after the partition, computational overhead for an existing member if the original group shrinks to a group of $x$ members), the original numbers of group members are 16, 32, 64, 128 respectively.

7.2.5 Merge Results. Merge costs are shown in Figure 16 when the resulting group size is 16, 32, 64, and 128. For a fixed number of resulting group size TGDH and BD show almost constant cost meaning that it does not depend on the number of current group members. In contrast, the performance of GDH strongly depends on the number of current group members, since the last member in the current group becomes the sponsor. 

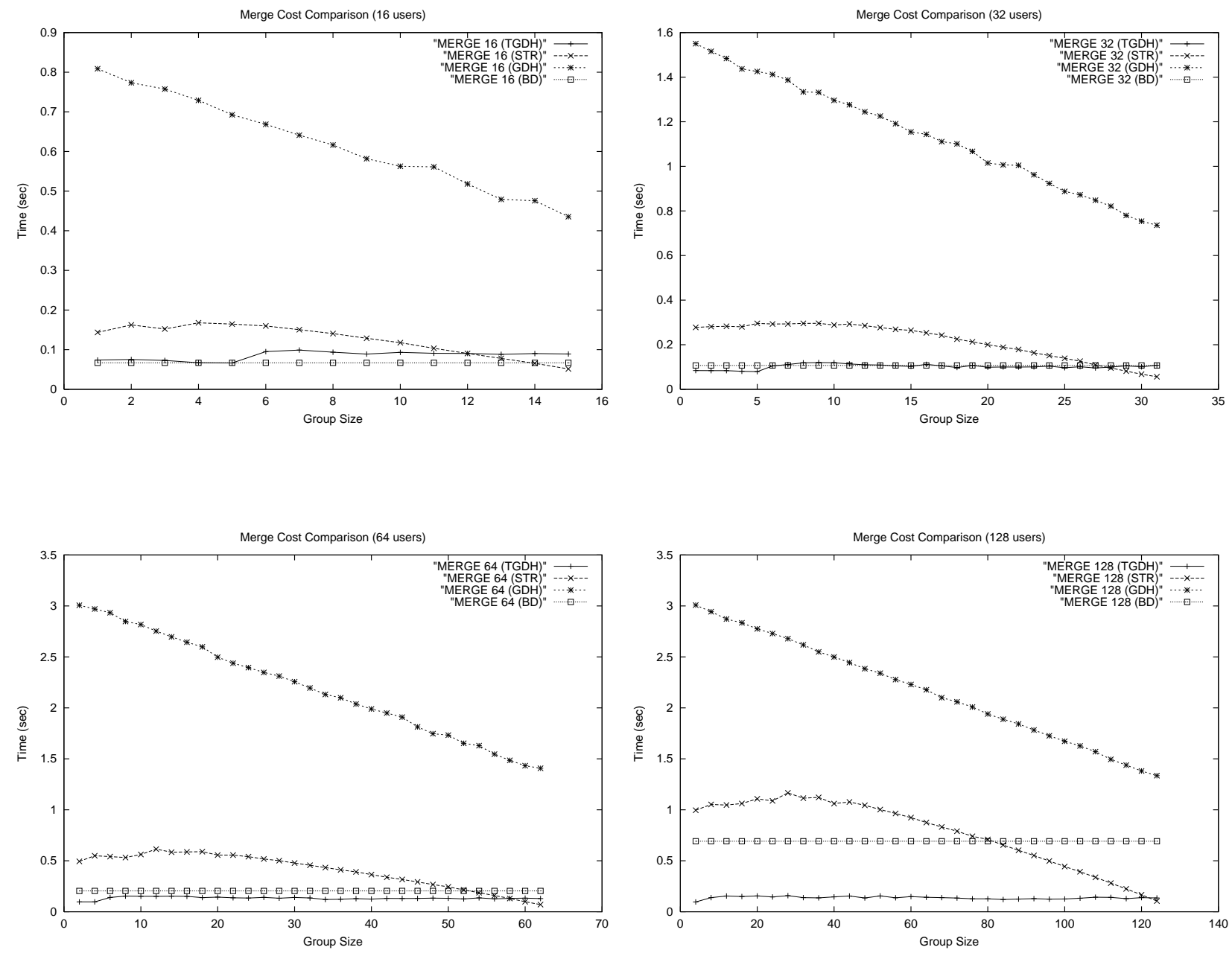

Fig. 16. Merge Cost Comparison: $(x, y)=$ (number of current group members, computational overhead for a member located in the group of $x$ members), after the membership event the number of group members becomes 16, 32, 64, and 128 respectively. 


\subsection{Discussion}

Based on the experimental results that measured the computational cost, TGDH exhibits the best performance despite the relatively high cost of partitions. Recall that, in practice, the self-clustering property of TGDH lessens the actual delay.

Looking at communication costs, it appears that TGDH also outperforms other protocols, except for partition events. In a high-delay WAN, the overall partition cost in TGDH may be high, although this effect can be lessened by self-clustering.

In high-delay WANs - where round-trip time exceeds $300 \mathrm{msec}$ - it is easy to see that computation cost for a small group is not so important. For example, if the group size is forty, the maximum difference in computational delay for a join is about $300 \mathrm{msec}$. In other words, communication costs (e.g. multicast vs. unicast, number of multicasts, number of rounds) are much more important in a high-delay network. Based on this consideration, the performance of STR gets better as communication delay increases. Overall, we conclude that TGDH performs best over low- and medium-delay networks.

\section{Related Work}

Group key management protocols come in three different flavors: contributory key agreement protocols, centralized, decentralized group key distribution scheme, and server-based key distribution protocols. Since the focus of this work is to provide common key to the dynamic peer group, we only consider the first two below.

\subsection{Group Key Agreement Protocols}

Research on group key agreement protocols started in 1982. We first summarize the early (theoretical) group key agreement protocols which did not consider dynamic membership operations; Most of them only supported group genesis.

The earliest contributory group key agreement built upon the 2-party Diffie-Hellman (DH) is due to Ingemarsson et al. (ING) [20]. In the fist round of ING, every member $M_{i}$ generates its session random $N_{i}$ and computes $\alpha^{N_{i}}$. In the subsequent rounds $k$ to $n-1, M_{i}$ computes $K_{i, k}=\left(K_{i-1 \bmod n, k-1}\right)^{N_{i}}$ where $K_{i-1}$ is the message received from $M_{i-1}$ in the previous round $k-1$. The resulting group key is of the form:

$$
K_{n}=\alpha^{N_{1} N_{2} N_{3} \ldots N_{n}} .
$$

The ING protocol is inefficient because: 1) every member has to start synchronously, 2) $n-1$ rounds are required to compute a group key, 3 ) it is hard to support dynamic membership operations due to its symmetry and 4) $n$ sequential modular exponentiations are required.

Another group key agreement developed for teleconferencing was proposed by Steer et al. [33]. This protocol is of particular interest since its group key structure is similar to that in TGDH.

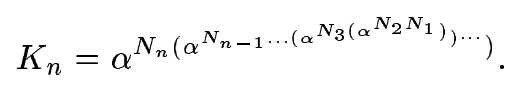

This protocol is well-suited for adding new group members as it takes only two rounds and four modular exponentiations. Member exclusion, however, is relatively difficult (for example, consider excluding $N_{1}$ from the group key).

Burmester and Desmedt construct an efficient protocol (called BD) which takes only two rounds and three modular exponentiations per member to generate a group key [14]. This efficiency allows all members to re-compute the group key for any membership change by rerunning the protocol. However, according to [34], most (at least half) of the members need to change their session random on every membership event. The group key in this protocol is different from STR and TGDH:

$$
K_{n}=\alpha^{N_{1} N_{2}+N_{2} N_{3}+\ldots+N_{n} N_{1}} .
$$

One shortcoming of $\mathrm{BD}$ is the high communication overhead. It requires $2 n$ broadcast messages and each member needs to generate 2 signatures and verify $2 n$ signatures. BD also has a hidden cost mentioned in Section 7.2. 
Becker and Wille analyze the minimal communication complexity of contributory group key agreement in general [8] and propose two protocols: octopus and hypercube. Their group key has the same structure as the key in TGDH. For example, for eight users their group key is:

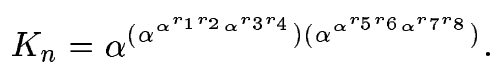

The Becker/Wille protocols handle join and merge operations efficiently, but the member leave operation is inefficient. Also, the hypercube protocol requires the group to be of size $2^{n}$ (for some integer $n$ ); otherwise, the efficiency slips.

Asokan et al. look at the problem of small-group key agreement, where the members do not have previously set up security associations [5]. Their motivating example is a meeting where the participants want to bootstrap a secure communication group. They adapt password authenticated DH key exchange to the group setting. Their setting, however, is different from ours, since they assume that all members share a secret password, whereas we assume a PKI where each member can verify any other members authenticity and authorization to join the group.

Tzeng and Tzeng propose an authenticated key agreement scheme that is based on secure multi-party computation [35]. This scheme also uses $2 n$ broadcast messages. Although the cryptographic mechanisms are quite elegant, a shortcoming is that the resulting group key does not provide perfect forward secrecy (PFS). If a long-term secret key is leaked, all previous and future group keys become insecure.

Steiner et al. first address dynamic membership issues [7,34] in group key agreement and propose a family of Group Diffie Hellman (GDH) protocols based on straight-forward extensions of the two-party Diffie-Hellman. GDH provides contributory authenticated key agreement, key independence, key integrity, resistance to known key attacks, and perfect forward secrecy. The GDH protocol suite is fairly efficient in leave and partition operation, but the merge protocol requires as many rounds as the number of new members to complete key agreement.

Perrig extends one-way function trees (OFT, originally introduced by McGrew and Sherman [24]) to design a tree-based key agreement scheme for peer groups [27]. This served as foundation for the design of our protocol.

TGDH also resembles OFT by some degree. One can claim that TGDH only modifies OFT by using $f(x)=\alpha^{x}$ $(\bmod p)$. This is, by part, true. However, there are major differences between OFT and TGDH: 1) Blinded key in OFT should not be revealed. However, all blinded keys in TGDH are public. Therefore, no secure channel is required. 2) TGDH is distributed, while OFT is centralized. The former requires no centralized entity who trasports secret keys. 3) OFT provides only join and leave operation. Furthermore its join policy is different from that of TGDH. 4) The security proof is essentially different. 5) Other contributions include robustness against cascaded event and self-clustering effect.

\subsection{Decentralized Group Key Distribution Protocols}

Decentralized group key distribution (DGKD) protocols involve dynamically selecting a group member who generates and distributes keys to other group members. After subtractive membership events, individual partitions can continue operation by electing a new key server. The drawback is that a key server must establish long-term pairwise secure channels (by making use of public key cryptosystem such as Diffie-Hellman) with all current group members in order to distribute group keys. Consequently, each time a new key server comes into play, significant costs must be incurred to set up these channels. Another disadvantage is the reliance on a single entity to generate good (i.e., cryptographically strong and random) keys.

First DGKD protocol is due to Waldvogel et al. [16]. They propose efficient protocols for small-group key agreement and large-group key distribution. Unfortunately, their scheme for autonomous small group key agreement is insecure (not collusion resistant).

Dondeti et al. modified OFT (One-way Function Tree) [24] to provide dynamic server election [18]. This protocol has the same key tree structure and uses the notations (e.g. keys, blinded keys) similar to ours. Other than expensive maintenance of secure channels described above, this protocol has a high communication cost: even for single join and leave, this protocol can take $O(h)$ rounds. This scheme does not handle merge and partition event. One advantage different from other DGKD protocols is that their group key has a contributory nature: whenever a group member changes its session random, the group key changes.

Rodeh et al. [30] propose a DGKD protocol derived from the LKH protocol [37]. It tolerates network partitions and other network events. Even though this approach does not avoid the disadvantages discussed above, it reduces the communication and computational costs. In addition, it uses AVL tree to provide provable tree height bound. 


\subsection{Other Related Work}

We can find further related work in the context of fault-tolerant computing and implementation issues. Protocol toolkits such as Rampart[28, 29] are designed to provide high degree of fault-tolerance, even in the presence of malicious (i.e., Byzantine) faults inside a group. However, these methods are expensive since they need reliable and atomic multicast secure against Byzantine faults.

Another interesting related work is due to Carman et al. [15]. This work compares energy consumption of group key agreement/distribution protocols in a sensor network by computer simulation. They point out that centralized group key distribution scheme is not appropriate for sensor network environments, though its power consumption is lower than group key agreement scheme. GDH and BD are compared with their group key management algorithm. It would be interesting to measure the power consumption of TGDH and STR protocol, but this is not our concern.

\section{Conclusion}

This paper presented a novel decentralized group key management approach, TGDH. In doing so, we unified two important trends in group key management: 1) key trees to efficiently compute and update group keys and 2) group Diffie-Hellman key exchange to achieve provably secure and fully distributed protocols. This yielded a secure, surprisingly simple and very efficient key management solution, which is supported, respectively, by the security arguments and the experiments. Moreover, our solution is inherently robust by virtue of being able to cope with cascaded (nested) key management operations which can stem from tightly spaced group membership changes. We believe this to be an issue of independent interest.

\section{References}

1. 5th ACM Conference on Computer and Communications Security, San Francisco, California, Nov. 1998. ACM Press.

2. Y. Amir. Replication using Group Communication over a Partitioned Network. PhD thesis, Institute of Computer Science, The Hebrew University of Jerusalem, Jerusalem, Israel, 1995.

3. Y. Amir, G. Ateniese, D. Hasse, Y. Kim, C. Nita-Rotaru, T. Schlossnagle, J. Schultz, J. Stanton, and G. Tsudik. Secure group communication in asynchronous networks with failures: Integration and experiments. In ICDCS 2000, Apr. 2000.

4. Y. Amir and J. Stanton. The spread wide area group communication system. Technical Report 98-4, Johns Hopkins University Department of Computer Science, 1998.

5. N. Asokan and P. Ginzboorg. Key-agreement in ad-hoc networks. In Nordsec'99, 1999.

6. N. Asokan, V. Shoup, and M. Waidner. Optimistic fair exchange of digital signatures. IEEE Journal on Selected Area in Communications, 18(4):593-610, 2000.

7. G. Ateniese, M. Steiner, and G. Tsudik. Authenticated Group Key Agreement and Friends. In ACMCCS98 [1], pages 17-26.

8. C. Becker and U. Wille. Communication complexity of group key distribution. In ACMCCS98 [1].

9. M. Bellare and P. Rogaway. Random oracles are practical: A paradigm for designing efficient protocols. In 1st ACM Conference on Computer and Communications Security, 1993.

10. D. Boneh. The Decision Diffie-Hellman problem. In Third Algorithmic Number Theory Symposium, number 1423 in Lecture Notes in Computer Science, pages 48-63. Springer-Verlag, Berlin Germany, 1998.

11. D. Boneh. Twenty years of attacks on the RSA cryptosystem. Notices of the American Mathematical Society (AMS), 46(2):203$213,1999$.

12. E. Bresson, O. Chevassut, and D. Pointcheval. Provably authenticated group Diffie-Hellman key exchange - the dynamic case. In C. Boyd, editor, Advances in Cryptology - ASIACRYPT '2001, Lecture Notes in Computer Science, Gold Coast, Australia, 2001. International Association for Cryptologic Research, Springer-Verlag, Berlin Germany.

13. E. Bresson, O. Chevassut, D. Pointcheval, and J.-J. Quisquater. Provably authenticated group diffie-hellman key exchange. In P. Samarati, editor, 8th ACM Conference on Computer and Communications Security, Philadelphia, PA, USA, Nov. 2001. ACM Press.

14. M. Burmester and Y. Desmedt. A secure and efficient conference key distribution system. In A. D. Santis, editor, Advances in Cryptology - EUROCRYPT '94, number 950 in Lecture Notes in Computer Science, pages 275-286. International Association for Cryptologic Research, Springer-Verlag, Berlin Germany, 1995. Final version of proceedings.

15. D. W. Carman, P. S. Kruss, and B. J. Matt. Constraints and approaches for distributed sensor network security. NAI Lab Technical Report 00-010, Network Assoiciates, Inc, September 2000.

16. G. Caronni, M. Waldvogel, D. Sun, N. Weiler, and B. Plattner. The VersaKey framework: Versatile group key management. IEEE Journal on Selected Areas in Communications, 17(9), Sept. 1999. 
17. D. Chaum. Zero-knowledge undeniable signatures. In I. Damgard, editor, Advances in Cryptology - EUROCRYPT ' 90 , number 473 in Lecture Notes in Computer Science, pages 458-464. Springer-Verlag, Berlin Germany, May 1991.

18. L. Dondeti, S. Mukherjee, and A.Samal. Disec: A distributed framework for scalable secure many-to-many communication. In Proceedings of The Fifth IEEE Symposium on Computers and Communications (ISCC 2000), July 2000.

19. A. Fekete, N. Lynch, and A. Shvartsman. Specifying and using a partionable group communication service. In $A C M P O D C$ '97, pages 53-62, Santa Barbara, CA, August 1997.

20. I. Ingemarsson, D. T. Tang, and C. K. Wong. A conference key distribution system. IEEE Transactions on Information Theory, 28(5):714-720, Sept. 1982.

21. Y. Kim, A. Perrig, and G. Tsudik. Simple and fault-tolerant key agreement for dynamic collaborative groups. In S. Jajodia, editor, 7th ACM Conference on Computer and Communications Security, pages 235-244, Athens, Greece, Nov. 2000. ACM Press.

22. Y. Kim, A. Perrig, and G. Tsudik. Communication-efficient group key agreement. In Information Systems Security, Proceedings of the 17th International Information Security Conference IFIP SEC'01, 2001.

23. A. K. Lenstra and E. R. Verheul. Selecting cryptographic key sizes. http://www. cryptosavvy . com/, Nov. 1999. Shorter version of the report appeared in the proceedings of the Public Key Cryptography Conference (PKC2000) and in the Autumn '99 PricewaterhouseCoopers CCE newsletter. To appear in Journal of Cryptology.

24. D. A. McGrew and A. T. Sherman. Key establishment in large dynamic groups using one-way function trees. Manuscript, May 1998.

25. L. Moser, Y. Amir, P. Melliar-Smith, and D. Agarwal. Extended virtual synchrony. In ICDCS '94, pages 56-65, June 1994.

26. OpenSSL Project team. Openssl, May 2001. http://www.openssl.org/.

27. A. Perrig. Efficient collaborative key management protocols for secure autonomous group communication. In International Workshop on Cryptographic Techniques and E-Commerce (CrypTEC '99), July 1999.

28. M. K. Reiter. Secure agreement protocols: Reliable and atomic group multicast in rampart. In J. Stern, editor, 2nd ACM Conference on Computer and Communications Security, pages 68-80, Fairfax, Virginia, Nov. 1994. ACM Press.

29. M. K. Reiter. A secure group membership protocol. 22(1):31-42, Jan. 1996.

30. O. Rodeh, K. Birman, and D. Dolev. Optimized rekey for group communication systems. In NDSS2000, pages 37-48, 2000.

31. V. Shoup. Lower bounds for discrete logarithms and related problems. In W. Fumy, editor, Advances in Cryptology - EUROCRYPT '97, number 1233 in Lecture Notes in Computer Science, pages 256-266. International Association for Cryptologic Research, Springer-Verlag, Berlin Germany, 1997.

32. V. Shoup. Using hash functions as a hedge against chosen ciphertext attacks. In B. Preneel, editor, Advances in Cryptology - EUROCRYPT '2000, number 1807 in Lecture Notes in Computer Science, pages 275-288. International Association for Cryptologic Research, Springer-Verlag, Berlin Germany, 2000.

33. D. Steer, L. Strawczynski, W. Diffie, and M. Wiener. A secure audio teleconference system. In S. Goldwasser, editor, Advances in Cryptology - CRYPTO '88, number 403 in Lecture Notes in Computer Science, pages 520-528, Santa Barbara, CA, USA, 1990. International Association for Cryptologic Research, Springer-Verlag, Berlin Germany.

34. M. Steiner, G. Tsudik, and M. Waidner. Key agreement in dynamic peer groups. IEEE Transactions on Parallel and Distributed Systems, August 2000.

35. W.-G. Tzeng and Z.-J. Tzeng. Round-efficient conference-key agreement protocols with provable security. In Advances in Cryptology - ASIACRYPT '2000, Lecture Notes in Computer Science, Kyoto, Japan, December 2000. International Association for Cryptologic Research, Springer-Verlag, Berlin Germany.

36. D. Wallner, E. Harder, and R. Agee. Key management for multicast: Issues and architecture. Internet-Draft draft-wallner-keyarch-00.txt, June 1997.

37. C. Wong, M. Gouda, and S. Lam. Secure group communications using key graphs. In Proceedings of the ACM SIGCOMM '98 conference on Applications, technologies, architectures, and protocols for computer communication, pages 68-79, 1998. Appeared in ACM SIGCOMM Computer Communication Review, Vol. 28, No. 4 (Oct. 1998).

38. C. Wong, M. Gouda, and S. Lam. Secure group communications using key graphs. IEEE/ACM Trans. on Networking, 8(1):16$30,2000$. 


\section{A Security Proof}

This section proves the security of TGDH. We introduce the Decisional (binary) Tree Group Diffie-Hellman problem (DTGDH) and, in a specific group setting, prove that DTGDH problem is reducible to 2-party Decision Diffie-Hellman (DDH) problem. Later, in Sections (A.3 - A.4), this result is used to prove the security of entire TGDH protocol suite.

\section{A.1 2-party Decision Diffie-Hellman Problem}

Our proofs require a specific group $G$. In this section, we introduce the group $G$ and define the 2-party Decision Diffie-Hellman (DDH) problem on $G$.

Let $k$ be a security parameter and $n$ be an integer. All algorithm run in probabilistic polynomial time with $k$ and $n$ as inputs.

For concreteness, we consider a specific $G$ :

On input $k$, algorithm gen chooses at random a pair $(q, \alpha)$ where $q$ is a $2 k$-bit value ${ }^{9}$, and $q$ and $p=2 q+1$ are both prime. Before introducing $G$, we first consider a group $\widehat{G}$, which is a group of squares modulo prime $p$. This group can be explained more precisely as follows: Consider an element $\alpha$ which is a square of a primitive element $\widehat{\alpha}$ of multiplicative group $\mathbb{Z}_{p}^{*}$, i.e. $\alpha=\widehat{\alpha}^{2}$. (Without loss of generality, we may assume $\alpha<q$.) Then group $\widehat{G}$ can be represented as

$$
\widehat{G}=\left\{\alpha^{i} \bmod p \mid i \in[1, q]\right\} .
$$

An attractive variation of this group is to represent the elements by the integers from 0 to $q-1$. The group operation is slightly different: Let a function $f$ be defined as

$$
f(x)= \begin{cases}x & \text { if } x \leq q \\ p-x & \text { if } q<x<p .\end{cases}
$$

Using this $f$ function, we can introduce the group $G$ as

$$
G=\left\{f\left(\alpha^{i} \bmod p\right) \mid i \in \mathbb{Z}_{q}\right\}
$$

Group operation on group $G$ is defined as $a \cdot b=f(a \cdot b(\bmod p))$, where $a, b \in G$.

Proposition 3. Let $g(x)=\alpha^{x} \bmod p$. Then the function $f \circ g$ is a bijection from $\mathbb{Z}_{q}$ to $\mathbb{Z}_{q}$.

Proof. To see this, suppose $f \circ g(x)=f \circ g(y)$. Then this can be written and $f(X)=f(Y)$ where integer $X=$ $\alpha^{x} \bmod p$ and $Y=\alpha^{y} \bmod p$. Now we can have four different cases:

$-X \leq q, Y \leq q$ : In this case, $f(X)=X$ and $f(Y)=Y$ and hence $X=Y$. Now we have an equation $\widehat{\alpha}^{2(x-y)}=$ $1 \bmod p$. Since $\widehat{\alpha}$ is a generator for $\mathbb{Z}_{p}^{*}$, its order (i.e. $2 q$ ) has to divide $2(x-y)$. This implies that $q$ has to divide $x-y$ and finally $x=y$ since $0<x, y \leq q$.

$-X>q, Y>q$ : In this case, $f(X)=p-X$ and $f(Y)=p-Y$ and hence $X=Y$. Rests are same as above.

$-X \leq q, Y>q$ : This case is impossible, since $\left(\frac{X}{p}\right)=1$ and $\left(\frac{p-Y}{p}\right)=-1$ since $p \equiv 3 \bmod 4$ and $X=p-Y$. $-X>q, Y \leq q$ : This is also impossible by similar reasoning.

Therefore, $f \circ g$ is an injection. It is also a surjection, since the sizes of domain and co-domain are the same.

Proposition 4. When a distribution $r$ is uniform and random in $G, f \circ g(r)$ is still uniform and random in $G$, since $f \circ g$ is bijective.

Groups of this type are also considered by Chaum [17]. It is generally assumed that DDH is intractable in these groups [10]. More concretely, the 2-party Decision Diffie-Hellman assumption on group $G$ is that for all polynomial time attackers $\mathcal{A}$, for all polynomials $Q(k) \exists k_{0} \forall k>k_{0}$, for $X_{0}:=N_{1} N_{2}$ and $X_{1}:=N_{3}$ with $N_{1}, N_{2}, N_{3} \in \mathcal{R} G$ uniformly chosen, and for a random bit $b$, the following equation holds:

$$
\left|\operatorname{Prob}\left[\mathcal{A}\left(1^{k} ; G ; \alpha ; \alpha^{N_{1}} ; \alpha^{N_{2}} ; \alpha^{X_{b}}\right)=b\right]-1 / 2\right|<1 / Q(k)
$$




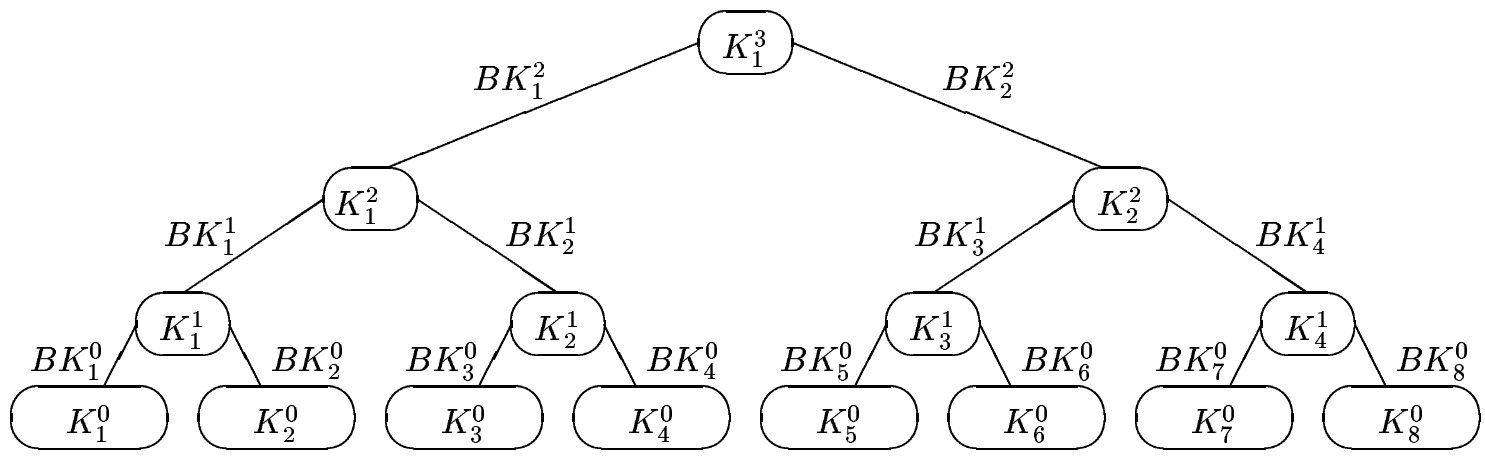

Fig. 17. Notations for fully balanced binary tree

\section{A.2 Decisional Binary Tree Group Diffie-Hellman Problem}

In this section we define the DTGDH problem (and assumption) and prove this problem is equivalent to 2-party decisional Diffie-Hellman problem. Figure 17 is an example of a key tree when $n=8$.

For $(q, \alpha) \leftarrow \operatorname{gen}(k), n \in \mathbb{N}$ and $X=\left(N_{1}, N_{2}, \ldots, N_{n}\right)$ for $N_{i} \in G$ and a key tree $T$ with $n$ leaf nodes which correspond to $N_{i}$, we define the following random variables:

- $K_{j}^{i}$ : $i$-th level $j$-th key (secret), each leaf node is associated with a member's session random, i.e. $K_{j}^{0}=N_{k}$ for some $k \in[1, n]$.

- $B K_{j}^{i}: i$-th level $j$-th blinded key (public), i.e. $\alpha^{K_{j}^{i}}$

$-K_{j}^{i}$ is recursively defined as follows:

$$
\begin{aligned}
K_{j}^{i} & =\alpha^{K_{2 j-1}^{i-1} K_{2 j}^{i-1}} \\
& =\left(B K_{2 j-1}^{i-1}\right)^{K_{2 j}^{i-1}} \\
& =\left(B K_{2 j}^{i-1}\right)^{K_{2 j-1}^{i-1}}
\end{aligned}
$$

In other words, we consider $K_{j}^{i}=f_{1}\left(N_{1}, N_{2}, \ldots, N_{n}\right)$ for some function $f_{1}$, and hence $B K_{j}^{i}=$ $\alpha^{f_{1}\left(N_{1}, N_{2}, \ldots, N_{n}\right)}=f_{2}\left(N_{1}, N_{2}, \ldots, N_{n}\right)$ for some function $f_{2}$.

For $(q, \alpha) \leftarrow \operatorname{gen}(k), n \in \mathbb{N}$ and $X=\left(N_{1}, N_{2}, \ldots, N_{n}\right)$ for $N_{i} \in G$ and a key tree $T$ with $n$ leaf nodes which correspond to $N_{i}$, we can define public and secret values collectively as below:

$$
\begin{aligned}
\text { view }(q, \alpha, h, X, T): & =\left\{B K_{j}^{i} \text { where } j \text { and } i \text { are defined according to } T\right\} \\
& =\left\{\alpha^{K_{j}^{i}} \bmod p \text { where } j \text { and } i \text { are defined according to } T\right\} \\
K(q, \alpha, h, X, T): & =\alpha^{K_{1}^{h-1} K_{2}^{h-1}}
\end{aligned}
$$

Since $(q, \alpha)$ are obvious from the context, we omit them in view () and $K()$. Note that view $(h, X, T)$ is exactly the view of the adversary in TGDH as will be described in Section 5, where the final secret key is $K(h, X, T)$. Let the following two random variables be defined by generating $(q, \alpha) \leftarrow \operatorname{gen}(k)$, choosing $X$ randomly from $G$ and choosing key tree $T$ randomly from all binary trees having $n$ leaf nodes:

- $A_{h}:=(v i e w(h, X, T), y)$

- $F_{h}:=(\operatorname{view}(h, X, T), K(h, X, T))$

Let the operator " $\approx_{\text {poly }}$ " denote polynomial indistinguishability.

\footnotetext{
${ }^{9}$ In order to achieve the security level $2^{-k}$, the group size should be at least $2^{2 k}$ [31].
} 
Proposition 5. [34] Let $K$ and $R$ be l-bit strings such that $R$ is a random and $K$ is a Diffie-Hellman key. We say that $K$ and $R$ are polynomially indistinguishable if, for all polynomial time distinguishers, $A$, the probability of distinguishing $K$ and $R$ is smaller than $\left(\frac{1}{2}+\frac{1}{Q(k)}\right)$, for all polynomial $Q(l)$.

Now we define DTGDH algorithm concretely:

Definition 6. Let $(q, \alpha) \leftarrow \operatorname{gen}(k), n \in \mathbb{N}$ and $X=\left(N_{1}, N_{2}, \ldots, N_{n}\right)$ for $N_{i} \in G$ and a key tree $T$ with $n$ leaf nodes which correspond to $N_{i}$, and $A_{h}$ and $F_{h}$ is defined as above. DTGDH algorithm $\mathcal{A}$ for group $G$ is a probabilistic polynomial time algorithm satisfying, for some fixed $k>0$ and sufficiently large $m$ :

$$
\left|\operatorname{Prob}\left[\mathcal{A}\left(A_{h}\right)=" \operatorname{True} "\right]-\operatorname{Prob}\left[\mathcal{A}\left(F_{h}\right)=" \operatorname{True} "\right]\right|>\frac{1}{m^{k}} .
$$

Accordingly, DTGDH problem is to find an Binary Tree DDH algorithm.

Now, we show that DTGDH problem is hard for the passive adversary: If the 2-party DDH in group $G$ defined above is hard, DTGDH problem is hard.

Using polynomial indistinguishability, the DTGDH problem defined in Definition 6 can be restated as: Find a polynomial distinguisher $\mathcal{A}$ which can distinguish $A_{h}$ and $F_{h}$ defined above.

Theorem 7. If the 2-party DDH on $G$ is hard, then $A_{h} \approx_{p o l y} F_{h}$.

Proof. We first note that $A_{h}$ and $F_{h}$ can be rewritten as:

If $X_{L}=\left(R_{1}, R_{2}, \ldots, R_{k}\right)$ and $X_{R}=\left(R_{k+1}, R_{k+2}, \ldots, R_{n}\right)$ where $R_{1}$ through $R_{k}$ are associated with leaf node in the left tree $T_{L}$ and $R_{k+1}$ through $R_{h}$ are in the right tree $T_{R}$ :

$$
\begin{aligned}
A_{h} & :=(\operatorname{view}(h, X, T), y) \quad \text { for random } y \in G \\
& =\left(\operatorname{view}\left(h-1, X_{L}, T_{L}\right), \operatorname{view}\left(h-1, X_{R}, T_{R}\right), B K_{1}^{h-1}, B K_{2}^{h-1}, y\right) \\
& =\left(\operatorname{view}\left(h-1, X_{L}, T_{L}\right), \operatorname{view}\left(h-1, X_{R}, T_{R}\right), \alpha^{K_{1}^{h-1}}, \alpha^{K_{2}^{h-1}}, y\right) \\
F_{h} & :=(\operatorname{view}(h, X), K(h, X)) \\
& =\left(\operatorname{view}\left(h-1, X_{L}, T_{L}\right), \operatorname{view}\left(h-1, X_{R}, T_{R}\right), B K_{1}^{h-1}, B K_{2}^{h-1}, \alpha^{K_{1}^{h-1} K_{2}^{h-1}}\right) \\
& =\left(\operatorname{view}\left(h-1, X_{L}, T_{L}\right), \operatorname{view}\left(h-1, X_{R}, T_{R}\right), \alpha^{K_{1}^{h-1}}, \alpha^{K_{2}^{h-1}}, \alpha^{K_{1}^{h-1} K_{2}^{h-1}}\right)
\end{aligned}
$$

We prove this theorem by induction and contradiction. The 2-party DDH problem in $G$ is equivalent to distinguishing $A_{1}$ and $F_{1}$. We assume that $A_{h-1}$ and $F_{h-1}$ are indistinguishable in polynomial time for the induction hypothesis. We further assume that there exists a polynomial algorithm that can distinguish between $A_{h}$ and $E_{h}$ for a random binary tree. We will show that this algorithm can be used to distinguish $A_{h-1}$ and $E_{h-1}$ or can be used to solve the 2-party DDH problem.

Consider the following:

$$
\begin{aligned}
& A_{h}:=\left(\operatorname{view}\left(h-1, X_{L}, T_{L}\right), \operatorname{view}\left(h-1, X_{R}, T_{R}\right), \alpha^{K_{L}^{h-1}}, \alpha^{K_{R}^{h-1}}, y\right) \\
& B_{h}:=\left(\operatorname{view}\left(h-1, X_{L}, T_{L}\right), \operatorname{view}\left(h-1, X_{R}, T_{R}\right), \alpha^{r}, \alpha^{K_{R}^{h-1}}, y\right) \\
& C_{h}:=\left(\operatorname{view}\left(h-1, X_{L}, T_{L}\right), \operatorname{view}\left(h-1, X_{R}, T_{R}\right), \alpha^{r}, \alpha^{r^{\prime}}, y\right) \\
& D_{h}:=\left(\operatorname{view}\left(h-1, X_{L}, T_{L}\right), \operatorname{view}\left(h-1, X_{R}, T_{R}\right), \alpha^{r}, \alpha^{r^{\prime}}, \alpha^{r r^{\prime}}\right) \\
& E_{h}:=\left(v i e w\left(h-1, X_{L}, T_{L}\right), \operatorname{view}\left(h-1, X_{R}, T_{R}\right), \alpha^{r}, \alpha^{K_{2}^{h-1}}, \alpha^{r K_{2}^{h-1}}\right) \\
& F_{h}:=\left(\operatorname{view}\left(h-1, X_{L}, T_{L}\right), \operatorname{view}\left(h-1, X_{R}, T_{R}\right), \alpha^{K_{L}^{h-1}}, \alpha^{K_{R}^{h-1}}, \alpha^{K_{L}^{h-1} K_{R}^{h-1}}\right)
\end{aligned}
$$

Since we can distinguish $A_{h}$ and $F_{h}$ in polynomial time, we can distinguish at least one of: $\left(A_{h}, B_{h}\right),\left(B_{h}, C_{h}\right)$, $\left(C_{h}, D_{h}\right),\left(D_{h}, E_{h}\right)$, or $\left(E_{h}, F_{h}\right)$. 
- $A_{h}$ and $B_{h}$ : Suppose we can distinguish $A_{h}$ and $B_{h}$ in polynomial time. We will show that this distinguisher $\mathcal{A}_{A B_{h}}$ can be used to solve DTGDH problem with height $h-1$. Suppose We want to decide whether $P_{h-1}^{\prime}=$ (view $\left.\left(h-1, X^{\prime}, T^{\prime}\right), r^{\prime}\right)$ is an instance of DTGDH problem or $r^{\prime}$ is a random number. To solve this, we generate another tree $T^{\prime \prime}$ of height $h-1$ with distribution $X^{\prime \prime}$. Note that we know all secret and public information of $T^{\prime \prime}$. Using $P_{h-1}^{\prime}$ and $\left(T^{\prime \prime}, X^{\prime \prime}\right)$, we generate a distribution:

$$
P_{h}^{\prime}=\left(\operatorname{view}\left(h-1, X^{\prime}, T^{\prime}\right), \operatorname{view}\left(h-1, X^{\prime \prime}, T^{\prime \prime}\right), \alpha^{r^{\prime}}, \alpha^{K\left(h-1, X^{\prime \prime}, T^{\prime \prime}\right)}, y\right)
$$

where $y \in_{\mathcal{R}} G$. Now we put $P_{h}^{\prime}$ as input of $\mathcal{A}_{A B_{h}}$. If $P_{h}^{\prime}$ is an instance of $A_{h}\left(B_{h}\right)$, then $P_{h-1}^{\prime}$ is an instance of $F_{h-1}\left(A_{h-1}\right)$ by Proposition 4.

- $B_{h}$ and $C_{h}$ : Suppose we can distinguish $B_{h}$ and $C_{h}$ in polynomial time. We will show that this distinguisher $\mathcal{A}_{B C_{h}}$ can be used to solve DTGDH problem with height $h-1$. Suppose We want to decide whether $P_{h-1}^{\prime}=($ view $(h-$ $\left.1, X^{\prime}, T^{\prime}\right), r^{\prime}$ ) is an instance of DTGDH problem or $r^{\prime}$ is a random number. To solve this, we generate another tree $T^{\prime \prime}$ of height $h-1$ with distribution $X^{\prime \prime}$ and choose $r^{\prime \prime} \in_{\mathcal{R}} G$. As before we know all secret and public information of $T^{\prime \prime}$. Using $P_{h-1}^{\prime}$ and $\left(T^{\prime \prime}, X^{\prime \prime}\right)$, we generate a distribution:

$$
P_{h}^{\prime}=\left(\operatorname{view}\left(h-1, X^{\prime \prime}, T^{\prime \prime}\right), \operatorname{view}\left(h-1, X^{\prime}, T^{\prime}\right), \alpha^{r^{\prime \prime}}, \alpha^{r^{\prime}}, y\right)
$$

where $y \in_{\mathcal{R}} G$. By Proposition 4, $r^{\prime \prime}$ is random and uniform in $G$. Now we put $P_{h}^{\prime}$ as input of $\mathcal{A}_{B C_{h}}$. If $P_{h}^{\prime}$ is an instance of $B_{h}\left(C_{h}\right)$, then $P_{h-1}^{\prime}$ is an instance of $F_{h-1}\left(A_{h-1}\right)$ by Proposition 4.

$-C_{h}$ and $D_{h}$ : Suppose we can distinguish $C_{h}$ and $D_{h}$ in polynomial time. Then, this distinguisher $\mathcal{A}_{C D_{h}}$ can be used to solve the 2-party DDH problem in group $G$. Note that $\alpha^{r}, \alpha^{r^{\prime}}$ are independent variable from view $(h-$ $\left.1, X_{L}, T_{L}\right)$ and view $\left(h-1, X_{R}, T_{R}\right)$. Suppose we want to test whether $\left(\alpha^{a}, \alpha^{b}, \alpha^{c}\right)$ is a DDH triple or not. To solve this, we generate two key trees $T_{1}$ and $T_{2}$ of height $h-1$ with distributions $X_{1}$ and $X_{2}$, respectively. Now we generate a new distribution:

$$
P_{h}^{\prime}=\left(\operatorname{view}\left(h-1, X_{1}, T_{1}\right), \operatorname{view}\left(h-1, X_{2}, T_{2}\right), \alpha^{a}, \alpha^{b}, \alpha^{c}\right) .
$$

If $P_{h}^{\prime}$ is an instance of $C_{h}\left(D_{h}\right)$, then $\left(\alpha^{a}, \alpha^{b}, \alpha^{c}\right)$ is a valid (invalid) DDH triple.

- $D_{h}$ and $E_{h}$ : Suppose we can distinguish $D_{h}$ and $E_{h}$ in polynomial time. We argue that this distinguisher $\mathcal{A}_{D E_{h}}$ can be used to solve DTGDH problem with height $h-1$. Suppose We want to decide if $P_{h-1}^{\prime}=(v i e w(h-$ $\left.1, X^{\prime}, T^{\prime}\right), r^{\prime}$ ) is an instance of DTGDH problem or $r^{\prime}$ is a random number. To solve this, we generate another tree $T^{\prime \prime}$ of height $h-1$ with distribution $X^{\prime \prime}$. As before, we know all secret and public information of $T^{\prime \prime}$. Using $P_{h-1}^{\prime}$ and $\left(T^{\prime \prime}, X^{\prime \prime}\right)$, we generate a distribution:

$$
\begin{aligned}
P_{h}^{\prime} & =\left(\operatorname{view}\left(h-1, X^{\prime}, T^{\prime}\right), \operatorname{view}\left(h-1, X^{\prime \prime}, T^{\prime \prime}\right), \alpha^{r^{\prime}}, \alpha^{r^{\prime \prime}},\left(\alpha^{r^{\prime}}\right)^{r^{\prime \prime}}\right) \\
& =\left(\operatorname{view}\left(h-1, X^{\prime}, T^{\prime}\right), \operatorname{view}\left(h-1, X^{\prime \prime}, T^{\prime \prime}\right), \alpha^{r^{\prime}}, \alpha^{r^{\prime \prime}}, \alpha^{r^{\prime} r^{\prime \prime}}\right)
\end{aligned}
$$

where $r^{\prime \prime} \in_{\mathcal{R}} G$. Since we generate $r^{\prime \prime}$, we can compute $\left(\alpha^{r^{\prime}}\right)^{r^{\prime \prime}}$. Now we put $P_{h}^{\prime}$ as input of $\mathcal{A}_{D E_{h}}$. If $P_{h}^{\prime}$ is an instance of $D_{h}\left(E_{h}\right)$, then $P_{h-1}^{\prime}$ is an instance of $F_{h-1}\left(A_{h-1}\right)$ by Proposition 4.

- $E_{h}$ and $F_{h}$ : Suppose we can distinguish $E_{h}$ and $F_{h}$ in polynomial time. We will show that this distinguisher $\mathcal{A}_{E F_{h}}$ can be used to solve DTGDH problem with height $h-1$. Suppose we want to decide if $P_{h-1}^{\prime}=($ view $(h-$ $\left.\left.1, X^{\prime}, T^{\prime}\right), r^{\prime}\right)$ is an instance of DTGDH problem or $r^{\prime}$ is a random number. To solve this, we generate another tree $T^{\prime \prime}$ of height $h-1$ with distribution $X^{\prime \prime}$. Again, we know all secret and public information of $T^{\prime \prime}$. Using $P_{h-1}^{\prime}$ and $\left(T^{\prime \prime}, X^{\prime \prime}\right)$, we generate a distribution:

$$
\begin{aligned}
P_{h}^{\prime} & =\left(\operatorname{view}\left(h-1, X^{\prime}, T^{\prime}\right), \operatorname{view}\left(h-1, X^{\prime \prime}, T^{\prime \prime}\right), \alpha^{r^{\prime}}, \alpha^{K\left(h-1 . X^{\prime \prime}, T^{\prime \prime}\right)},\left(\alpha^{r^{\prime}}\right)^{K\left(h-1 . X^{\prime \prime}, T^{\prime \prime}\right)}\right) \\
& =\left(\operatorname{view}\left(h-1, X^{\prime}, T^{\prime}\right), \operatorname{view}\left(h-1, X^{\prime \prime}, T^{\prime \prime}\right), \alpha^{r^{\prime}}, \alpha^{K\left(h-1 . X^{\prime \prime}, T^{\prime \prime}\right)}, \alpha^{r^{\prime} K\left(h-1 . X^{\prime \prime}, T^{\prime \prime}\right)}\right)
\end{aligned}
$$

where $r^{\prime} \in_{\mathcal{R}} G$. Since $r^{\prime}$ is given, we can compute $\left(\alpha^{r^{\prime}}\right)^{K\left(h-1 . X^{\prime \prime}, T^{\prime \prime}\right)}$. Now we put $P_{h}^{\prime}$ as input of $\mathcal{A}_{E F_{h}}$. If $P_{h}^{\prime}$ is an instance of $E_{h}\left(F_{h}\right)$, then $P_{h-1}^{\prime}$ is an instance of $F_{h-1}\left(A_{h-1}\right)$ by Proposition 4. 


\section{A.3 Group Key Secrecy}

Before considering the group key secrecy, we briefly examine key freshness. Every group key is fresh, since at least one member in the group generates its random key share uniformly for every membership change ${ }^{10}$. The probability that new group key is same as any old group key is negligible due to bijectiveness of $(f \circ g)$ function.

We note that the root (group) key is never used directly for the purposes of encryption, authentication or integrity. Instead, special-purpose sub-keys are derived from the root key, e.g., by applying a cryptographically secure hash function to the root key, i.e. H(group key) is used for such applications.

As discussed in Section 3, decisional group key secrecy is more meaningful if sub-keys are derived from a group key. Decisional group key secrecy of TGDH protocol is somewhat related to DTGDH assumption mentioned in Section A.2. This assumption ensures that there is no information leakage other that public blinded key informations.

We can also derive the sub-keys based on Shoup's hedge [32]: Compute the key as $H$ (group key $) \oplus \mathcal{H}($ group key) where $\mathcal{H}$ is a random oracle. It follows that in addition to the security in the standard model based on DTGDH assumption, the derived key is also secure in the random oracle model [9] based on Computational Tree-based Group Diffie-Hellman assumption.

\section{A.4 Key Independence}

We now give an informal proof that TGDH satisfies forward and backward secrecy, or equivalently key independence. In order to show that TGDH provides key independence, we only need to show that the view of the former (prospective) member to the current tree is exactly same as the view of the passive adversary respectively, since this shows that the advantage of the former (prospective) member is same as the passive adversary and by Theorem 7 .

We first consider backward secrecy, which states that a new member who knows the current group key cannot derive any previous group key. Let $M_{n+1}$ be the new member. The sponsor for this join event changes its session random and, consequently, previous root key is changed. Therefore, the view of $M_{n+1}$ with respect to the prior key tree is exactly same as the view of an outsider. Hence, the new member does not gain any advantage compared to a passive adversary.

This argument can be easily extended to the merge of two or more groups. When a merge happens, sponsor in each tree changes its session random. Therefore, each member's view on other member's tree is exactly same as the view of a passive adversary. This shows that the newly merged member has exactly same advantage about any of the old key tree as a passive adversary.

Now we consider the forward secrecy, meaning that a passive adversary who knows a contiguous subset of old group keys cannot discover subsequent group keys. Here, we consider partition and leave at the same time. Suppose $M_{d}$ is a former group member. Whenever subtractive event happens, a sponsor refreshes its session random, and, therefore, all keys known to leaving members will be changed accordingly. Therefore, $M_{d}$ 's view is exactly same as the view of the passive adversary.

This proves that TGDH provides decisional version of key independence.

\footnotetext{
${ }^{10}$ Recall that insider attacks are not our concern. This excludes the case when an insider intentionally generates non-random number.
} 